\title{
THE INFLUENCE OF THERMAL PRESSURE ON EQUILIBRIUM MODELS OF HYPERMASSIVE NEUTRON STAR MERGER REMNANTS
}

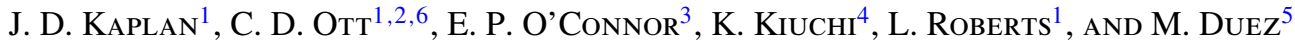 \\ ${ }^{1}$ TAPIR, California Institute of Technology, Mailcode 350-17, Pasadena, CA 91125, USA; cott@ tapir.caltech.edu \\ ${ }^{2}$ Kavli IPMU (WPI), University of Tokyo, Kashiwa, Japan \\ ${ }^{3}$ CITA, University of Toronto, 60 St. George Street, Toronto, ON M5S 3H8, Canada \\ ${ }^{4}$ Yukawa Institute for Theoretical Physics, University of Kyoto, Kyoto, Japan \\ ${ }^{5}$ Department of Physics and Astronomy, Washington State University, Pullman, WA, USA \\ Received 2013 June 17; accepted 2014 June 1; published 2014 June 27
}

\begin{abstract}
The merger of two neutron stars leaves behind a rapidly spinning hypermassive object whose survival is believed to depend on the maximum mass supported by the nuclear equation of state (EOS), angular momentum redistribution by (magneto-)rotational instabilities, and spindown by gravitational waves. The high temperatures $(\sim 5-40 \mathrm{MeV})$ prevailing in the merger remnant may provide thermal pressure support that could increase its maximum mass and, thus, its life on a neutrino-cooling timescale. We investigate the role of thermal pressure support in hypermassive merger remnants by computing sequences of spherically symmetric and axisymmetric uniformly and differentially rotating equilibrium solutions to the general-relativistic stellar structure equations. Using a set of finite-temperature nuclear EOS, we find that hot maximum-mass critically spinning configurations generally do not support larger baryonic masses than their cold counterparts. However, subcritically spinning configurations with mean density of less than a few times nuclear saturation density yield a significantly thermally enhanced mass. Even without decreasing the maximum mass, cooling and other forms of energy loss can drive the remnant to an unstable state. We infer secular instability by identifying approximate energy turning points in equilibrium sequences of constant baryonic mass parameterized by maximum density. Energy loss carries the remnant along the direction of decreasing gravitational mass and higher density until instability triggers collapse. Since configurations with more thermal pressure support are less compact and thus begin their evolution at a lower maximum density, they remain stable for longer periods after merger.
\end{abstract}

Key words: dense matter - equation of state - stars: neutron

Online-only material: color figures

\section{INTRODUCTION}

Coalescing double neutron stars (NSs) are prime candidate progenitors of short-hard gamma-ray bursts (GRBs; e.g., Nakar 2007 and references therein). The strong gravitational wave emission driving the coalescence makes NSNS systems the primary targets of the network of second-generation gravitationalwave interferometers currently under construction (Advanced LIGO: Harry et al. 2010; Advanced Virgo: Accadia et al. 2011; and KAGRA: Somiya et al. 2012).

Until the last moments of inspiral, the constituent NSs may essentially be treated as cold NSs. Tidal heating is mild and the NS crust may not fail until the NSs touch (Penner et al. 2012, but see Tsang et al. 2012; Weinberg et al. 2013). Merger results in the formation of a shocked, extremely rapidly differentially spinning central object, commonly referred to as a hypermassive NS (HMNS), since it comprises the vast majority of the baryonic mass of the two premerger NSs and is thus expected to be more massive than the maximum mass supported by the nuclear equation of state (EOS) in the spherical and uniformly rotating limits limit (see, e.g., Faber \& Rasio 2012 for a review of NSNS mergers). The subsequent evolution of the HMNS has important ramifications for gravitational wave emission and the possible transition to a short-hard GRB. If the HMNS survives for an extended period, nonaxisymmetric rotational instability may enhance the high-frequency gravitational-wave emission, possibly allowing gravitational-wave observers to constrain the

\footnotetext{
6 Alfred P. Sloan Research Fellow.
}

nuclear EOS (e.g., Bauswein et al. 2012). On the other hand, the neutrino-driven wind blown off a surviving HMNS, producing mass loss at a rate of order $10^{-4} M_{\odot} \mathrm{s}^{-1}$, will lead to strong baryon loading in polar regions (Dessart et al. 2009), making the formation of the relativistic outflows needed for a GRB more difficult, even if a black hole with an accretion disk forms eventually. If the HMNS collapses to a black hole within milliseconds of merger, baryon loading will not hamper a GRB, but strong gravitational-wave and neutrino emission would be shut off rapidly.

The long-term survival of the HMNS depends sensitively on the maximum mass of a nonrotating cold NS supported by the nuclear EOS, which most certainly is above $\sim 2 M_{\odot}$ (Demorest et al. 2010; Antoniadis et al. 2013) and very likely below $\sim 3.2 M_{\odot}$ (Lattimer \& Prakash 2007). At its formation, the HMNS is rapidly and strongly differentially rotating. Extreme differential rotation alone may increase the maximum HMNS mass by more than $100 \%$ (e.g., Baumgarte et al. 2000). Angular momentum redistribution by (magneto-)rotational instabilities and spindown by gravitational wave emission are expected to remove this additional support. This will ultimately lead to black hole formation if the HMNS mass is above the maximum mass that can be supported by the nuclear EOS and uniform rotation $(\lesssim 20 \%$ greater than the maximum in the nonrotating limit; Baumgarte et al. 2000).

Recently, Sekiguchi et al. (2011), Paschalidis et al. (2012), Bauswein et al. (2010), and, in earlier work, Baiotti et al. (2008), have argued that thermal pressure support at moderately high temperatures of $\sim 5-40 \mathrm{MeV}$ (Oechslin et al. 2007; Sekiguchi 
et al. 2011) may significantly influence the structure and evolution of the postmerger HMNS and prolong its lifetime until collapse to a black hole. If true, the HMNS may survive on the neutrino cooling timescale provided that the combined premerger mass of the NSs is sufficiently close to the thermally enhanced maximum HMNS mass. These authors estimate the neutrino cooling timescale to be comparable to or longer than the timescale for angular momentum redistribution and spindown by gravitational waves.

The focus of this paper is on the role of thermal pressure support in HMNS merger remnants. Postmerger HMNS configurations that survive for multiple dynamical times quickly assume dynamical equilibrium and, after the extremely dynamic merger phase, show only mild deviation from axisymmetry (e.g., Sekiguchi et al. 2011; Shibata et al. 2005). Hence, instead of performing computationally expensive full merger simulations, we investigate the role of thermal effects by approximating HMNS configurations as sequences of rotational equilibrium solutions, which we compute with the relativistic self-consistent field method (Komatsu et al. 1989a, 1989b; Cook et al. 1992). We consider the spherical limit (Tolman-Oppenheimer-Volkoff (TOV) solutions), uniform, and differential rotation. We employ multiple finite-temperature microphysical nuclear EOS and, since the equilibrium solver requires a barotropic EOS, a range of temperature and composition parameterizations that are motivated by the merger simulations of Sekiguchi et al. (2011). An overall similar approach, though only considering isothermal and isentropic configurations, has been used in the past to study thermal effects on uniformly and differentially rotating proto-NSs (Goussard et al. 1997, 1998).

The key quantity relevant in the secular evolution of HMNSs is the baryonic mass ( $M_{\mathrm{b}}$; also called "rest mass") that can be supported by a given combination of EOS, thermal/ compositional structure, and rotational setup. The gravitational mass $\left(M_{\mathrm{g}}\right)$ is not conserved and is reduced by cooling and angular momentum loss. Our results show that the maximum baryonic mass of TOV, uniformly rotating, and differentially rotating configurations is essentially unaffected by thermal pressure support. Thermal pressure support is negligible at supranuclear densities and becomes significant only at densities below nuclear saturation density. Since maximum-mass configurations always have maximum and mean densities above nuclear, thermal pressure support is minimal. The thermal contribution to the stress-energy tensor (which sources curvature) may, depending on the EOS, even lead to a net decrease of the $M_{\mathrm{b}}^{\max }$ with increasing temperature.

We find thermal enhancement of $M_{\mathrm{b}}$ for configurations with mean densities less than a few times nuclear saturation density that are nonrotating or rotating subcritically (i.e., below the mass-shedding limit). A hot configuration in this regime will support the same baryonic mass at a lower mean (and maximum) density. However, hot rotating configurations are spatially more extended than their cold counterparts, and thus reach mass shedding at lower angular velocities. This counteracts the thermal enhancement and results in $M_{\mathrm{b}}^{\max }$ that are within a few percent of cold configurations.

The secular evolution of an HMNS toward collapse is driven by energy losses to gravitational waves and neutrinos, and, potentially, by loss of angular momentum transported to the surface by processes such as the magnetorotational instability (MRI). It proceeds along trajectories of constant (or nearly constant) baryonic mass and in the direction of decreasing total energy (i.e., gravitational mass $M_{\mathrm{g}}$ ) and increasing maximum baryon density $\rho_{\mathrm{b}, \max }$ (i.e., more compact configurations). We conjecture, based on established results of the theory of rotating relativistic stars (Friedman \& Stergioulas 2013), that instability to collapse occurs when the configuration reaches an unstable part of the parameter space and not necessarily because the maximum supportable baryonic mass $M_{\mathrm{b}}^{\max }$ drops below $M_{\mathrm{b}}$. We formalize this via an approximate variant of the turning-point theorem (e.g., Sorkin 1982; Friedman \& Stergioulas 2013): The turning-point theorem states that for uniformly rotating NSs, a local extremum in $M_{\mathrm{g}}$ at fixed angular momentum, entropy, and baryonic mass constitutes a point at which secular instability to collapse must set in. We argue that the turning point theorem carries over to differentially rotating hot HMNSs. The precise turning points become approximate and are distributed over a narrow range of $\rho_{\mathrm{b}, \max }$ and $M_{\mathrm{g}}$ for all degrees of differential rotation and temperature prescriptions that we consider here. The regime of instability is thus largely independent of HMNS temperature. However, a hotter configuration will be less compact initially and, hence, will begin its secular evolution to its turning point at a lower $\rho_{\mathrm{b}, \max }$ than a colder one. It will thus have to evolve further until it reaches its turning point and, at a fixed rate of energy loss, will survive for longer.

This paper is structured as follows. In Section 2, we introduce the set of EOS we employ and discuss the relative importance of thermal pressure as a function of density. We also introduce the temperature and composition parameterizations and the methods used for constructing equilibrium models without and with rotation. In Section 3, we lay out our results for nonrotating NSs and then discuss uniformly and differentially rotating configurations in Sections 4.1 and 4.2, respectively. We consider evolutionary sequences of HMNSs at constant baryonic mass in the context of an approximate turning point theorem and compare with results from recent merger simulations in Section 5. Finally, in Section 6, we summarize our results and conclude.

\section{METHODS AND EQUATIONS OF STATE}

\subsection{Equations of State}

We use a set of eight EOS in this study. All EOS produce cold NSs in $\beta$-equilibrium that can have gravitational masses $M_{\mathrm{g}}$ above $2 M_{\odot}$. These include two EOS from Lattimer \& Swesty (1991), the $K_{0}=220 \mathrm{MeV}$ and $K_{0}=375 \mathrm{MeV}$ variants (where $K_{0}$ is the nuclear compressibility modulus), denoted LS220 and LS375; the relativistic mean field (RMF) model EOS from Shen et al. (2011c), denoted HShen; two RMF models based on the NL3 and the FSUGold parameter set (Shen et al. 2011a, 2011b) denoted GShen-NL3 and GShen-FSU2.1; an unpublished ${ }^{7}$ RMF model based on the DD2 interaction denoted HSDD2; and two recent RMF model EOS fit to astrophysical measurements of NS masses and radii (Steiner et al. 2013), denoted SFHo and SFHx. All of these EOS are available in a common format for download from http://www.stellarcollapse.org.

The EOS of finite-temperature nuclear matter in nuclear statistical equilibrium (NSE) has contributions from a baryonic component (nucleons and nuclei), a relativistic electron/ positron Fermi gas, a photon gas, and, if neutrinos are trapped, a neutrino gas. The Helmholtz free energies of these components add linearly, and the pressure is then the sum of the partial pressures and a function of baryon density $\rho$, temperature $T$, and electron fraction $Y_{e}$,

\footnotetext{
7 Available from http://phys-merger.physik.unibas.ch/ hempel/eos.html, based on Hempel et al. (2012) and Hempel \& Schaffner-Bielich (2010).
} 


$$
P=P_{\text {baryon }}+P_{e}+P_{\gamma}+P_{\nu} \text {. }
$$

While $P_{\text {baryon }}$ varies between the employed EOS, we add $P_{e}$ and $P_{\gamma}$ using the Timmes EOS (Timmes \& Arnett 1999) available from http://cococubed.asu.edu. In hot HMNSs, like in protoNSs, neutrinos are trapped and in equilibrium with matter. We include their pressure contribution to the EOS by treating them as a noninteracting relativistic Fermi gas with chemical potential $\mu_{v_{i}}$. For a single species of neutrinos and antineutrinos, the neutrino pressure in equilibrium is

$$
P_{v_{i}}=\frac{4 \pi\left(k_{B} T\right)^{4}}{3(h c)^{3}}\left[F_{3}\left(\eta_{v_{i}}\right)+F_{3}\left(-\eta_{v_{i}}\right)\right] \times \exp \left(-\frac{\rho_{\text {trap }}}{\rho}\right),
$$

where $\eta_{v_{i}}=\mu_{v_{i}} /\left(k_{B} T\right)$ is the neutrino degeneracy parameter. For HMNS conditions, all neutrino species are present, but $v_{\mu}$ and $v_{\tau}$ have $\mu_{v_{i}}=0$, since they appear only in particle-antiparticle pairs that have equal and opposite chemical potentials. For electron neutrinos we use $\mu_{v_{e}}=\mu_{e}+\mu_{p}-\mu_{n}$, for electron antineutrinos we use $\mu_{\bar{v}_{e}}=-\mu_{v_{e}}$. We include an attenuation factor $\exp \left(-\rho_{\text {trap }} / \rho\right)$ to account for the fact that neutrinos decouple from matter at low densities. We set $\rho_{\text {trap }}=$ $10^{12.5} \mathrm{~g} \mathrm{~cm}^{-3}$, which is a fiducial trapping density for protoNSs (e.g., Liebendörfer 2005). Taking the exact expression for the difference of the Fermi integrals from Bludman \& van Riper (1978), we have the total neutrino pressure summed over all three species,

$$
\begin{aligned}
P_{v}= & \frac{4 \pi\left(k_{B} T\right)^{4}}{3(h c)^{3}}\left[\frac{21 \pi^{4}}{60}+\frac{1}{2} \eta_{v_{e}}^{2}\left(\pi^{2}+\frac{1}{2} \eta_{v_{e}}^{2}\right)\right] \\
& \times \exp \left(-\frac{\rho_{\text {trap }}}{\rho}\right) .
\end{aligned}
$$

We note that due to the neutrino statistical weight $g=1$, for a single species of relativistic non-degenerate $v-\bar{v}$ pairs, the pressure is a factor of two lower than for $e^{-}-e^{+}$pairs, since $e^{-}$ and $e^{+}$have statistical weight (spin degeneracy) 2 .

Figure 1 illustrates the contributions of the partial pressures to the total pressure as a function of baryon density $\rho_{\mathrm{b}}$ for neutron-rich HMNS matter at two temperatures, $0.5 \mathrm{MeV}$ (a representative "cold" temperature) and $20 \mathrm{MeV}$ (a representative "hot" temperature for HMNSs). For the $0.5 \mathrm{MeV}$ EOS, we set the electron fraction $Y_{e}$ by solving for $\nu$-less $\beta$-equilibrium $\left(\mu_{v_{e}}=0\right)$. The resulting EOS describes ordinary cold NSs (at $0.5 \mathrm{MeV}$ any thermal effects are negligible). For the $20 \mathrm{MeV}$ case, we solve for $Y_{e}$ by assuming $\nu$-full $\beta$-equilibrium. We do so by making the assumption that any neutrinos produced during the merger are immediately trapped in the HMNS core, but stream away from regions below trapping density. The procedure is discussed in the next Section 2.2 and detailed in Appendix B.

Near and above nuclear saturation density, $\rho_{\text {nuc }} \simeq 2.6 \times$ $10^{14} \mathrm{~g} \mathrm{~cm}^{-3}$ for the LS220 EOS, the baryon pressure is due to the repulsive core of the nuclear force and dominates in both cold and hot regimes. The thermal enhancement above $\rho_{\text {nuc }}$ remains small even at $20 \mathrm{MeV}$. In the cold case, relativistically degenerate electrons $\left(\Gamma=(d \ln P)(d \ln \rho)^{-1}=4 / 3\right)$ dominate below $\rho_{\text {nuc }}$. At $20 \mathrm{MeV}$, relativistic non-degenerate electron/positron pairs and photons (for both, $P \propto T^{4}$, independent of $\rho_{\mathrm{b}}$; see, e.g., van Riper \& Bludman 1977) are the primary contributors at low densities, while the baryon pressure is significantly thermally enhanced below nuclear saturation density and dominates above $\sim 10^{12} \mathrm{~g} \mathrm{~cm}^{-3}$. The neutrino pressure is comparable to the

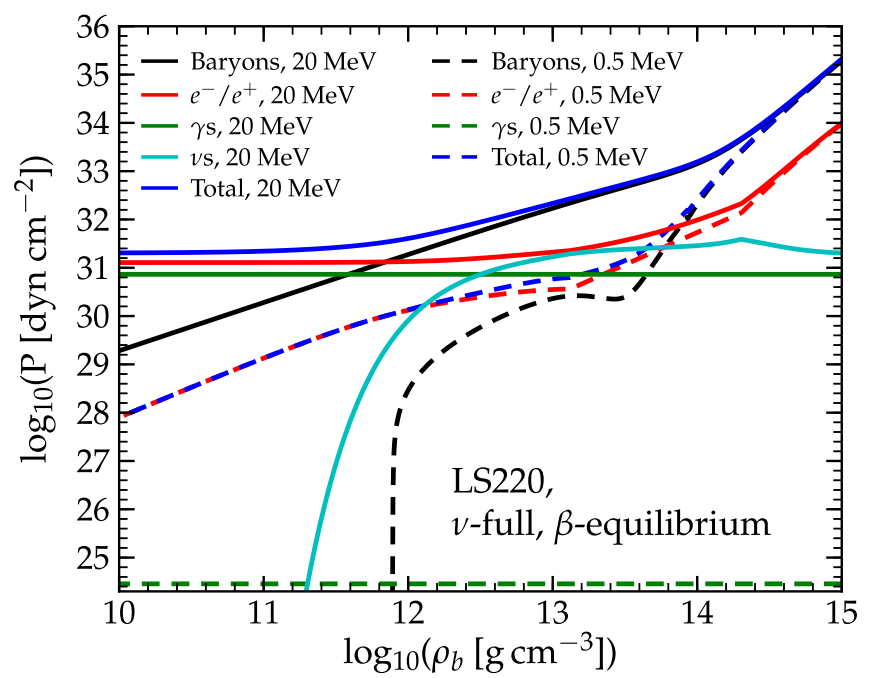

Figure 1. Individual pressure contributions of baryons, electrons/positrons, photons, and trapped neutrinos and the total pressure as a function of baryon density in the LS220 EOS for $\nu$-full $\beta$-equilibrium as described in the text and $T=0.5 \mathrm{MeV}$ (dashed lines) and $T=20 \mathrm{MeV}$ (solid lines). The qualitative and quantitative behavior of the LS220 EOS with increasing temperature is representative for all EOS considered in this study. Note that the baryon pressure becomes negative at $\rho_{\mathrm{b}} \lesssim 10^{12} \mathrm{~g} \mathrm{~cm}^{-3}$, and dips around $10^{13.5} \mathrm{~g} \mathrm{~cm}^{-3}$ due to Coulomb effects at low temperatures (Lattimer \& Swesty 1991).

(A color version of this figure is available in the online journal.)

degenerate electron pressure between $\sim 10^{12.5}$ and $10^{14} \mathrm{~g} \mathrm{~cm}^{-3}$, but still subdominant to the nuclear component. The contribution of pairs and photons gradually becomes more important at all densities as the temperature increases. We note that for $T=0.5 \mathrm{MeV}$, the neutrino chemical potentials are all zero and the pressure of trapped neutrinos is $3 \times(7 / 8) \times P_{\gamma}$, thermodynamically insignificant at $T=0.5 \mathrm{MeV}$.

\subsection{Temperature and Composition Parameterizations}

The hydrostatic and rotational equilibrium equations that we solve in this study assume a barotropic EOS $(P=P(\rho))$ and do not provide constraints on thermal structure and composition ( $Y_{e}$ is the only relevant compositional variable in NSE). We must make some assumptions to be able to proceed and obtain $P=P\left(\rho, T(\rho), Y_{e}(\rho, T(\rho))\right)$ for our general finite-temperature microphysical EOS. Old NSs in isolation are nearly isothermal (e.g., Prakash et al. 2001) and so are coalescing NSs until tidal heating becomes significant (e.g., Kochanek 1992; Lai 1994). During merger, the NS matter is shock-heated to tens of MeV and results of the few merger simulations that have been carried out with temperature-dependent EOS (e.g., Sekiguchi et al. 2011; Bauswein et al. 2010; Oechslin et al. 2007; Rosswog \& Liebendörfer 2003; Ruffert \& Janka 2001) indicate that the HMNS is far from being isothermal or isentropic. It has a very hot dense core with $T \sim 20-40 \mathrm{MeV}$ surrounded by a lower-density cooler envelope/torus of 5-20 MeV, which may also be almost Keplerian and, hence, centrifugally supported. This result appears to be robust for equal-mass or near equalmass NSNS systems (which may dominate the population; e.g., Lattimer 2012 and references therein). Mergers of non-equal mass systems in which the lower-mass NS is tidally wrapped around its more massive companion reach similar temperatures, but generally tend to have more mass at lower densities in the disk/torus (Oechslin et al. 2007).

There is no unique model/EOS independent mapping $T=$ $T(\rho)$, thus we must explore a variety of possibilities. In 

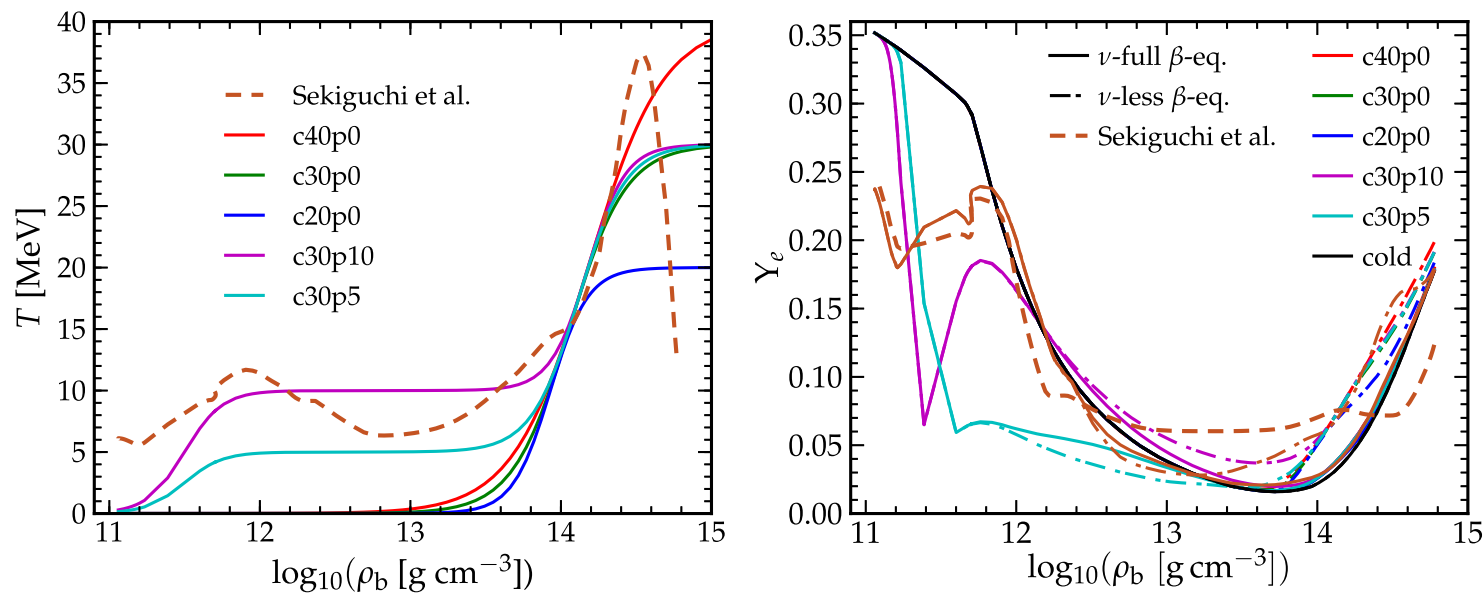

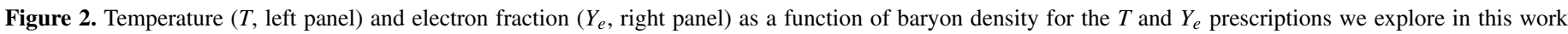

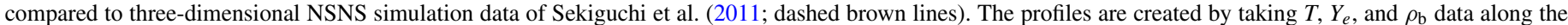

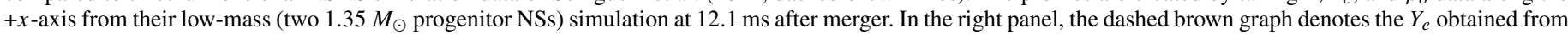
the simulation, while the solid and the dash-dotted graphs are $Y_{e}$ obtained from the simulation temperature profile for $\nu$-full and $\nu$-less $\beta$-equilibrium, respectively.

(A color version of this figure is available in the online journal.)

Figure 2, we contrast our set of temperature parameterizations with a $T(\rho)$ profile obtained from a $1.35-1.35 M_{\odot}$ simulation using the HShen EOS by Sekiguchi et al. (2011) at $\sim 12 \mathrm{~ms}$ after merger. We consider very hot cores at 20,30 , and $40 \mathrm{MeV}$ with cold envelopes (parameterizations c20p0, c30p0, and c40p0) and two parameterizations with very hot cores at $30 \mathrm{MeV}$ and cool envelopes at $10 \mathrm{MeV}$ and $5 \mathrm{MeV}, \mathrm{c} 30 \mathrm{p} 10$ and c30p5, respectively. Since low-density regions have shorter neutrino cooling times, the c30p10 and c30p5 parameterization may represent early HMNSs, while the cold-envelope parameterizations c20p0, c30p0, and c40p0 may correspond to late-time HMNSs. Note that the $\mathrm{c} 30 \mathrm{p} 10$ parameterization fits the temperature profile from the Sekiguchi et al. (2011) simulation quite well. Details on the functional forms of our parameterizations can be found in Appendix A. For the TOV case we also consider isothermal configurations as a limiting case.

The choice of $Y_{e}(\rho, T(\rho))$ is equally difficult. Before merger, the NSs are in $\nu$-less $\beta$-equilibrium $\left(\mu_{v}=\mu_{e}+\mu_{p}-\mu_{n}=0\right)$. After merger, neutrinos are present. They are trapped in hot dense matter $\left(\mu_{v} \neq 0\right)$ and are streaming away from low-density regions. The equilibrium $Y_{e}$ will shift and mixing due to nonlinear hydrodynamics in the HMNS phase will distort any initial $Y_{e}(\rho, T(\rho))$ profile.

We deem the following prescription for $Y_{e}$ to be the physically most sensible. We assume that the NSNS merger occurs so rapidly that the electron fraction $Y_{e}$ of the $\nu$-less $\beta$-equilibrium in the NSs becomes the trapped postmerger lepton fraction $Y_{\text {lep }}=Y_{e}+Y_{\nu_{e}}-Y_{\bar{v}_{e}}$ above $\rho_{\text {trap }}$. Using the $\beta$-equilibrium condition with nonzero $\mu_{v}$, we solve for $Y_{e}$. At densities below $\rho_{\text {trap }}$ we transition to $Y_{e}$ given by $\nu$-less $\beta$-equilibrium. Details of this procedure are given in Appendix B. We refer to this parameterization of $Y_{e}$ as $\nu$-full $\beta$-equilibrium. In addition and for comparison, we consider choices of constant $Y_{e}=0.1$ and $Y_{e}$ set according to $\nu$-less $\beta$-equilibrium. We note that our parameterization of $Y_{e}$ is ad hoc and cannot account for mixing and neutrino transport effects in the merger process. The right panel of Figure 2 depicts $Y_{e}(\rho, T(\rho))$ as obtained from the simulation of Sekiguchi et al. (2011) contrasted with $Y_{e}$ profiles computed under the assumption of $v$-less and $v$-full $\beta$-equilibrium for various temperature parameterizations and for the $T(\rho)$ as given by the simulation. None of the prescriptions fit the simulation- $Y_{e}$ particularly well, which indicates that mixing and neutrino transport effects are important (but cannot be included here). The $Y_{e}$ obtained using the temperature data from the simulation naturally fits best, in particular at low densities where neutrinos have decoupled from the matter and $\nu$-less $\beta$ equilibrium holds.

In the top panels of Figure 3, we show the fractional pressure increase due to thermal effects as a function of baryon density for our set of temperature parameterizations for the LS220 EOS (left panel) and the HShen EOS (right panel) as two representative example EOS. We also distinguish between the choices of $Y_{e}$ parameterization. For the parameterizations with cold "mantles" (cXp0), thermal effects are most important at densities near $\sim \rho_{\text {nuc }}$ and quickly lose significance at lower and higher densities in both EOS. The thermal pressure enhancement is at most a factor of three (for the HShen) to five (for the LS220 EOS) for these parameterizations. The situation is different for the cases with hot plateaus, c30p10 and c30p5. For these, the thermal pressure is up to 20 times larger at low densities than predicted by the cold EOS. The $Y_{e}$ parameterizations corresponding to $v$-full and $\nu$-less $\beta$-equilibrium yield qualitatively and quantitatively very similar results for both EOS.

At low densities, the $\nu$-full and $\nu$-less $\beta$-equilibrium cases both lead to $Y_{e}>0.1$ (see Figure 2). As a consequence, the pressure in the unrealistic $Y_{e}=$ const. $=0.1, \mathrm{cXp} 0$ parameterizations is lower than in the cold $v$-less case at $\rho_{\mathrm{b}} \lesssim 10^{12.2} \mathrm{~g} \mathrm{~cm}^{-3}$. Due to the logarithmic scale of Figure 3, the graphs of $\mathrm{cXp} 0$ with $Y_{e}=0.1$ start only there and the predicted pressure enhancement is higher than in the $\beta$-equilibrium cases, which lead to lower $Y_{e}$ above $\sim 10^{12.2} \mathrm{~g} \mathrm{~cm}^{-3}$ and below $\sim \rho_{\text {nuc }}$. In the cases with hot plateau (c30p10 and c30p5), thermal effects dominate over differences in $Y_{e}$ at low densities. Finally, at $\rho>\rho_{\text {nuc }}$, where temperature effects are smaller, differences in $Y_{e}$ become important. Since the nuclear component dominates there, lower $Y_{e}$ corresponds to higher pressure (e.g., Lattimer \& Prakash 2001) and both $\beta$-equilibrium cases yield $Y_{e}>0.1$.

The lower panels of Figure 3 depict the relative contribution of the neutrinos to the total (hot) pressure in the HMNS temperature and $Y_{e}$ parameterizations considered in this study. While there are clear temperature (see Equation (3)) and $Y_{e}$ (through $\mu_{v_{e}}$ ) dependences, neutrino pressure plays only a minor role, making 

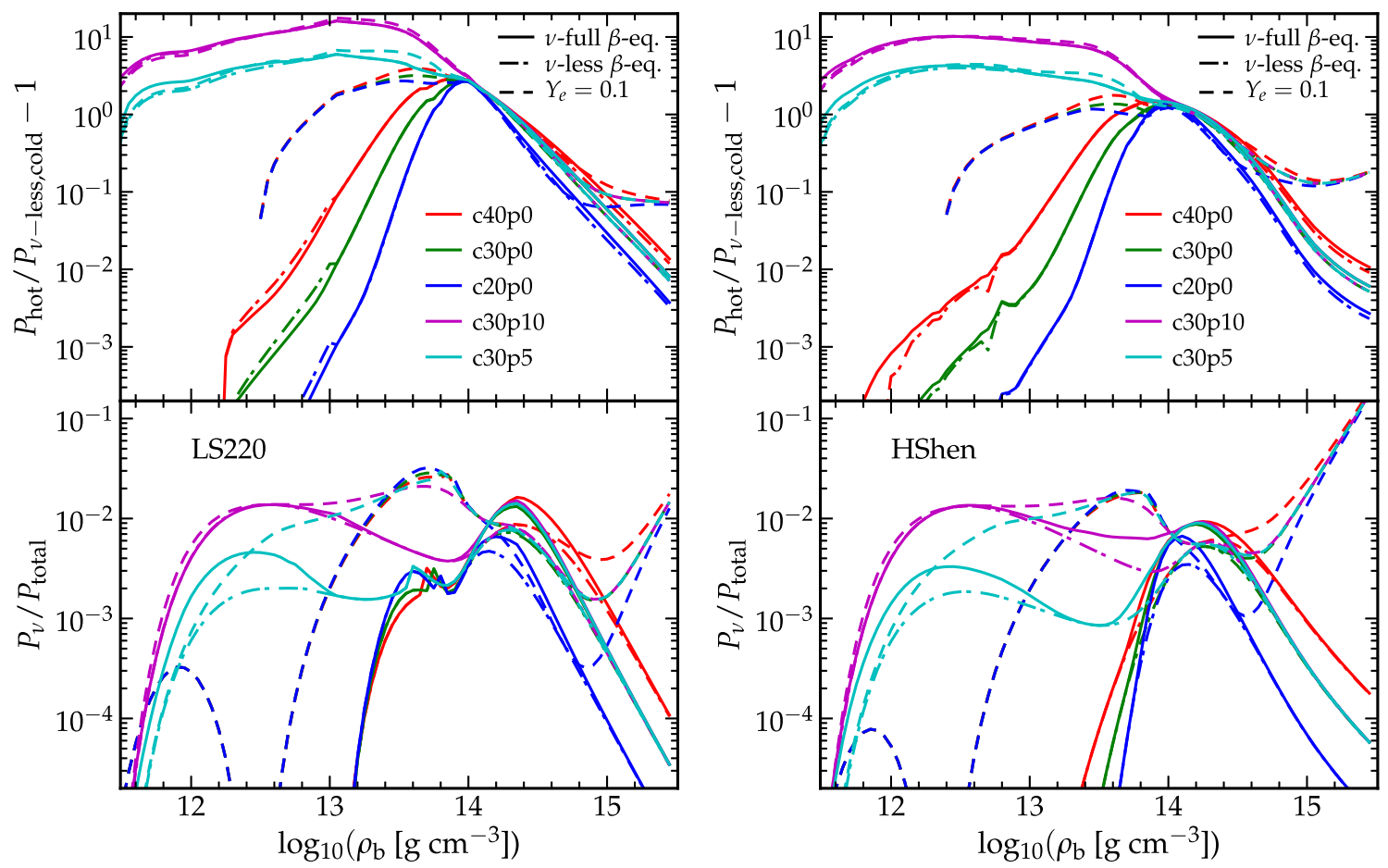

Figure 3. Effects of temperature and $Y_{e}$ parameterizations on the pressure and relevance of the neutrino pressure component. Top panels: fractional increase of the pressure over the cold $\nu$-less $\beta$-equilibrium pressure for the LS220 EOS (left panel) and the HShen EOS (right panel). The different line styles correspond to $Y_{e}(\rho)$ obtained in $v$-full $\beta$-equilibrium (solid), $v$-less $\beta$-equilibrium (dash-dotted), and constant $Y_{e}=0.1$ (dashed). Bottom panels: relative contribution of the neutrinos to the total pressure (see Equation (3)) in the five temperature and three $Y_{e}$ parameterizations and the LS220 EOS (left panel) and the HShen EOS (right panel).

(A color version of this figure is available in the online journal.)

up at most $\sim 2 \%$ of the total pressure of the LS220 EOS. This is true also for the HShen EOS with the exception of the unrealistic $Y_{e}=0.1$ case in which the neutrino pressure contribution grows to $\gtrsim 10 \%$ of the total pressure at supranuclear densities.

Finally, we note that the temperature and $Y_{e}$ prescriptions discussed here lead to regions that may be unstable to convection if not stabilized by a positive specific angular momentum gradient (e.g., Tassoul 1978). The spherically and axially symmetric equilibrium models that we construct in this study cannot account for convection and we leave an analysis of convective instability to future work.

\subsection{Spherically Symmetric Equilibrium Models}

We solve the TOV equation (e.g., Shapiro \& Teukolsky 1983),

$$
\begin{aligned}
\frac{d P}{d r}= & \left.-\frac{G}{r^{2}}\left[\rho_{\mathrm{b}}\left(1+\frac{\epsilon}{c^{2}}+\frac{P}{\rho_{\mathrm{b}} c^{2}}\right)\right] M_{\mathrm{g}}(r)+4 \pi r^{3} \frac{P}{c^{2}}\right] \\
& \times\left[1-\frac{2 G M_{\mathrm{g}}(r)}{r c^{2}}\right]^{-1},
\end{aligned}
$$

where $r$ is the areal (circumferential) radius, $\rho_{\mathrm{b}}$ is the baryon density, $\epsilon$ is the specific internal energy, and $M_{\mathrm{g}}(r)$ is the gravitational mass enclosed by radius $r$, determined via

$$
\frac{d M_{\mathrm{g}}}{d r}=4 \pi r^{2} \rho_{\mathrm{b}}\left[1+\frac{\epsilon}{c^{2}}\right] .
$$

The baryonic mass is larger and given by

$$
\frac{d M_{\mathrm{b}}}{d r}=4 \pi r^{2} \rho_{\mathrm{b}}\left(1-\frac{2 G M_{\mathrm{g}}(r)}{r c^{2}}\right)^{-1 / 2} .
$$

We construct the TOV solutions using a standard fourthorder Runge-Kutta integrator on an equidistant grid with $\delta R=$
$10^{2} \mathrm{~cm}$ zones. After each integration sub-step, the EOS $P=$ $P\left(\rho_{\mathrm{b}}\right)$ is inverted to obtain $\rho_{\mathrm{b}}$. We use a variety of $P\left(\rho_{\mathrm{b}}\right)$ parameterizations: (1) $T=$ const. (isothermal) with $v$-full $\beta$-equilibrium above $\rho_{\text {trap }}$ and $\nu$-less $\beta$-equilibrium below, (2) $T=$ const. with $\nu$-less $\beta$-equilibrium, (3) $T=$ const. with constant $Y_{e}=0.1$, and (4) the phenomenological $\mathrm{cXpX}$ temperature parameterizations with $\nu$-full $\beta$-equilibrium above $\rho_{\text {trap }}$ and $\nu$-less equilibrium below. We compute TOV solutions for all EOS and define the surface of the NS as the areal radius at which one of the following two conditions is true: (1) the pressure equals $10^{-10}$ of the central pressure; (2) the pressure predicted by the integration of Equation (4) drops below the lowest pressure value available in the equation of state table. The latter is not a limitation, because the high-density TOV configurations considered here have steep density and pressure profiles near their surfaces. The pressure dropping to very small values thus indicates that the surface has been reached.

Besides the EOS, temperature, and $Y_{e}$ prescription, the central baryon density $\rho_{\mathrm{b}, c}$ is the only other free parameter. Since we are interested in the maximum mass that can be supported, we compute sequences with varying $\rho_{\mathrm{b}, \mathrm{c}}$ for each EOS, but limit ourselves to $\rho_{\text {nuc }}<\rho_{\mathrm{b}, \mathrm{c}} \leqslant \rho_{\max , \mathrm{EOS}}$, where the latter is just the maximum density entry in the respective EOS table. HMNSs with central densities below $\rho_{\text {nuc }}$ are not realistic (see Sekiguchi et al. 2011).

We make our TOV solver, all $P=P\left(\rho_{\mathrm{b}}\right)$ tables, and the Python scripts used to create the results in this paper available on http://www.stellarcollapse.org.

\subsection{Axisymmetric Equilibrium Models}

We generate axisymmetric equilibrium models using the code originally presented in (Cook et al. 1992, hereafter CST; see 
also Cook et al. 1994a, 1994b), which is based on the relativistic self-consistent field method of Komatsu et al. (1989a). The axisymmetric equilibrium equations are solved iteratively on a grid in $(s, \mu)$, where $s$ is a compactified radial coordinate and $\mu=\cos \theta$, where $\theta$ is the usual spherical polar angle. Additionally, metric functions are solved using Green's functions integrals expanded in terms of $N_{l}$ Legendre polynomials. Consequently, the total numerical resolution is specified via a tuple of $\left(N_{s}, N_{\mu}, N_{l}\right)$, which we set to $(500,300,16)$. The resolution is chosen so that the resulting integral quantities of the equilibrium solution (e.g., its gravitational mass) are precise to about one part in $10^{3}$. The surface of the star is defined by an enthalpy contour which is specified in the code by setting a surface energy density. This energy density has a default value of $7.9 \mathrm{~g} \mathrm{~cm}^{-3}$, and we have checked that increasing its value by a factor of $10^{6}$ leaves the physical quantities of the solution unchanged to our stated general error level of $10^{-3}$.

An axisymmetric HMNS equilibrium configuration is constructed by the CST code based on choices of (1) a barotropic EOS, (2) a rotation law, (3) the rotation rate, and, (4) the maximum mass-energy density $E_{\max }=\left[\rho_{\mathrm{b}}\left(1+\epsilon / c^{2}\right)\right]_{\max }$ of the configuration.

In order to keep the size of the parameter space manageable, we restrict rotating configurations to the LS220 and HShen EOS and set up barotropic versions using the temperature and composition parameterizations described in Section 2.2. Since the EOS obtained with $\nu$-full and $\nu$-less $\beta$-equilibrium differ only very mildly (see Figure 3 ), we construct rotating configurations under the simple assumption of $\nu$-less $\beta$-equilibrium.

We employ the " $j-$ const." rotation law (see, e.g., CST), which is commonly used in the literature for HMNS models (e.g., Baumgarte et al. 2000). The degree of differential rotation is parameterized by $\tilde{A} .^{8}$ In the Newtonian limit, this rotation law becomes $\Omega=\Omega_{c} /\left(1+\tilde{A}^{2} \varpi^{2} / r_{\mathrm{e}}^{2}\right)$, where $\varpi$ is the cylindrical radius, $r_{\mathrm{e}}$ is the radius of the star at its equator, and $\Omega_{c}$ is the central angular velocity. For $\tilde{A}=0$, one recovers uniform rotation, while for large $\tilde{A}$, the specific angular momentum becomes constant (i.e., $\Omega \propto \varpi^{-2}$ in the Newtonian limit). We explore values of $\tilde{A}$ between 0 and 1 . The latter value of $\tilde{A}$ corresponds to roughly a factor of two decrease of the angular velocity from the center to the HMNS surface, which is in the ball park of what is found in merger simulations (e.g., Shibata et al. 2005). Once the rotation law is fixed, the rotation rate is determined by specifying the axis ratior $_{\mathrm{p} / \mathrm{e}}$, defined as the ratio of the HMNS radius along the pole $r_{\mathrm{p}}$ divided by the radius at the equator $r_{\mathrm{e}}$.

The final parameter to be chosen is the maximum energy density of the configuration. For simplicity and consistency with the choice of variables for the TOV solutions discussed in Section 2.3, we set $E_{\max }$ by choosing a maximum baryon density $\rho_{\mathrm{b}, \max }$ and obtain $E\left(\rho_{\mathrm{b}, \max }\right)$ from the EOS.

For each choice of EOS, $\rho_{\mathrm{b}, \max }$, and $\tilde{A}$, we compute a sequence of models with increasing rotation rate, stepping down from $r_{\mathrm{p} / \mathrm{e}}=1$ (the nonrotating TOV case) until we reach mass shedding or until the code fails to converge to an equilibrium solution. In the case of uniform rotation $(\tilde{A}=0)$ the sequence always ends at mass shedding, the resulting rotating NS has spheroidal shape, and the maximum and central density

\footnotetext{
8 Note that $\tilde{A}=1 / \hat{A}$, where $\hat{A}$ is the same $\hat{A}$ as used in Baumgarte et al.
} (2000). coincide $\left(\rho_{\mathrm{b}, \max }=\rho_{\mathrm{c}}\right)$. Differentially rotating sequences, on the other hand, can bifurcate into two branches: one with $\rho_{\mathrm{b}, \max }=\rho_{\mathrm{c}}$ and spheroidal geometry and one with an off-center location of $\rho_{\mathrm{b}, \max }$ and quasitoroidal shape. For differentially rotating models, the CST solver generally fails to converge to a solution at $r_{\mathrm{p} / \mathrm{e}}$ before mass shedding and, therefore, possibly before the maximum mass for a given configuration is reached. This limitation means that the maximum masses we state for differentially rotating models are to be interpreted as lower bounds on the true maximum masses. The code developed by Ansorg et al. (2003) is far more robust than CST for such extreme configurations and these authors have argued that with increasing degree of differential rotation, arbitrarily large masses could be supported in extremely extended tori, but such configurations are unlikely to be astrophysically relevant.

\section{RESULTS: SPHERICALLY SYMMETRIC MODELS}

Our main interest is in how temperatures in the range encountered in HMNS of NSNS postmerger simulations change the maximum mass that can be supported. Since baryonic mass is a conserved quantity and can be related to the number of baryons present in the individual NSNS before merger (modulo a small amount of potential ejecta), we treat it as a the most important variable and define the maximum gravitational masses $M_{\mathrm{g}}^{\max }$ as the gravitational mass at which $M_{\mathrm{b}}^{\max }$ is maximal. We consider the isothermal TOV solution as a limiting case of maximal thermal support but note that such configurations with $T \gtrsim 5-8 \mathrm{MeV}$ develop very large, non-degenerate envelopes at the low end of the central baryon densities $\rho_{\mathrm{b}, \mathrm{c}}$ considered here. With increasing temperature, degeneracy is more and more lifted at those densities and the TOV model approaches an isothermal sphere whose pressure is dominated by relativistic non-degenerate pairs and whose mass and radius become infinite. We discard such solutions.

The results of our TOV calculations are summarized by Figure 4 for all considered EOS. We provide numerical results in Table 1 for fiducial isothermal cold $(T=0.5 \mathrm{MeV})$ and parameterized temperature choices.

In the top panel of Figure 4, we show the maximum gravitational mass (defined as $M_{\mathrm{g}}$ at $M_{\mathrm{b}}^{\mathrm{max}}$ ) as a function of isothermal temperature for our three $Y_{e}$ prescriptions. The considered EOS show a great degree of variation in their sensitivity to $Y_{e}$ prescriptions, but the overall trend is clear: increasing temperature generally leads to increasing $M_{\mathrm{g}}^{\max }$. The fractional increase over the cold value, however, is not large, as shown by the center panel. The HShen and GShen-FSU2.1 RMF TOV stars are the most sensitive to temperature variations, ${ }^{9}$ but even their maximum gravitational TOV mass increases only by $\sim 12 \%-15 \%$ at isothermal $T=50 \mathrm{MeV}$. The cXpX temperature parameterizations, shown as symbols in Figure 4 located at their respective central temperatures, generally follow the trend of the isothermal sequences for each EOS, but their $M_{\mathrm{g}}^{\max }$ enhancement is systematically lower, since they are only centrally hot.

The lower panel of Figure 4 depicts the change of the maximum baryonic TOV mass $M_{\mathrm{b}}^{\max }$ with increasing temperature. For most EOS, $M_{\mathrm{b}}^{\max }$ stays roughly constant at low temperatures, but decreases at high temperatures. This shows that the increase in $M_{\mathrm{g}}^{\max }$ in the TOV solutions is primarily due to thermal contributions to the total mass-energy density. Since it is the mass-energy density, and not just the baryonic mass, which

\footnotetext{
9 See, e.g., Hempel et al. (2012) for a discussion of EOS physics and temperature dependence of various EOS models.
} 
Table 1

Summary of TOV Results for All EOS

\begin{tabular}{|c|c|c|c|c|c|}
\hline EOS & $T(\rho)$ & $\begin{array}{l}M_{\mathrm{b}}^{\max } \\
\left(M_{\odot}\right)\end{array}$ & $\begin{array}{l}M_{\mathrm{g}}^{\max } \\
\left(M_{\odot}\right)\end{array}$ & $\begin{array}{c}R \\
(\mathrm{~km})\end{array}$ & $\begin{array}{c}\rho_{c} \\
\left(10^{15} \mathrm{~g} \mathrm{~cm}^{-3}\right)\end{array}$ \\
\hline LS220, v-less & $0.5 \mathrm{MeV}$ & 2.406 & 2.042 & 10.63 & 1.863 \\
\hline \multirow{5}{*}{ LS220, v-full } & c20p0 & 2.434 & 2.068 & 10.69 & 1.873 \\
\hline & c30p0 & 2.433 & 2.078 & 10.89 & 1.840 \\
\hline & c30p10 & 2.433 & 2.079 & 11.86 & 1.840 \\
\hline & c30p5 & 2.433 & 2.078 & 11.23 & 1.840 \\
\hline & $\mathrm{c} 40 \mathrm{p} 0$ & 2.428 & 2.087 & 11.07 & 1.808 \\
\hline LS375, v-less & $0.5 \mathrm{MeV}$ & 3.349 & 2.715 & 12.34 & 1.243 \\
\hline \multirow[t]{5}{*}{ LS375, v-full } & c20p0 & 3.322 & 2.717 & 12.59 & 1.232 \\
\hline & c30p0 & 3.294 & 2.717 & 12.68 & 1.221 \\
\hline & c30p10 & 3.293 & 2.718 & 13.49 & 1.221 \\
\hline & c30p5 & 3.293 & 2.717 & 12.95 & 1.221 \\
\hline & $\mathrm{c} 40 \mathrm{p} 0$ & 3.264 & 2.714 & 12.75 & 1.210 \\
\hline HShen, v-less & $0.5 \mathrm{MeV}$ & 2.560 & 2.214 & 12.59 & 1.357 \\
\hline \multirow[t]{5}{*}{ HShen, $v$-full } & c20p0 & 2.584 & 2.246 & 13.17 & 1.321 \\
\hline & c30p0 & 2.601 & 2.273 & 13.48 & 1.276 \\
\hline & c30p10 & 2.604 & 2.277 & 15.08 & 1.276 \\
\hline & c30p5 & 2.603 & 2.275 & 14.01 & 1.276 \\
\hline & $\mathrm{c} 40 \mathrm{p} 0$ & 2.613 & 2.295 & 13.69 & 1.243 \\
\hline GShen-NL3, v-less & $0.5 \mathrm{MeV}$ & 3.353 & 2.765 & 13.34 & 1.115 \\
\hline \multirow[t]{5}{*}{ GShen-NL3, v-full } & c20p0 & 3.354 & 2.781 & 13.51 & 1.098 \\
\hline & c30p0 & 3.344 & 2.791 & 13.70 & 1.081 \\
\hline & c30p10 & 3.346 & 2.793 & 15.04 & 1.081 \\
\hline & c30p5 & 3.345 & 2.792 & 14.30 & 1.081 \\
\hline & $\mathrm{c} 40 \mathrm{p} 0$ & 3.330 & 2.796 & 13.86 & 1.070 \\
\hline GShen-FSU2.1, $v$-less & $0.5 \mathrm{MeV}$ & 2.468 & 2.114 & 11.67 & 1.505 \\
\hline \multirow{5}{*}{ GShen-FSU2.1, v-full } & c20p0 & 2.488 & 2.140 & 12.15 & 1.474 \\
\hline & c30p0 & 2.497 & 2.159 & 12.40 & 1.428 \\
\hline & c30p10 & 2.502 & 2.164 & 14.30 & 1.420 \\
\hline & c30p5 & 2.497 & 2.160 & 12.44 & 1.428 \\
\hline & $\mathrm{c} 40 \mathrm{p} 0$ & 2.504 & 2.176 & 12.56 & 1.398 \\
\hline HSDD2, v-less & $0.5 \mathrm{MeV}$ & 2.896 & 2.419 & 11.92 & 1.395 \\
\hline \multirow{5}{*}{ HSDD2, $v$-full } & c20p0 & 2.891 & 2.429 & 12.28 & 1.381 \\
\hline & $\mathrm{c} 30 \mathrm{p} 0$ & 2.883 & 2.436 & 12.43 & 1.367 \\
\hline & c30p10 & 2.884 & 2.437 & 13.47 & 1.367 \\
\hline & c30p5 & 2.883 & 2.436 & 12.79 & 1.367 \\
\hline & $\mathrm{c} 40 \mathrm{p} 0$ & 2.871 & 2.440 & 12.55 & 1.353 \\
\hline SFHo, $v$-less & $0.5 \mathrm{MeV}$ & 2.433 & 2.057 & 10.31 & 1.906 \\
\hline \multirow[t]{5}{*}{ SFHo, $v$-full } & c20p0 & 2.434 & 2.068 & 10.67 & 1.884 \\
\hline & $\mathrm{c} 30 \mathrm{p} 0$ & 2.433 & 2.078 & 10.86 & 1.862 \\
\hline & c30p10 & 2.434 & 2.079 & 11.81 & 1.862 \\
\hline & c30p5 & 2.433 & 2.078 & 11.21 & 1.851 \\
\hline & $\mathrm{c} 40 \mathrm{p} 0$ & 2.428 & 2.087 & 11.03 & 1.829 \\
\hline SFHx, $v$-less & $0.5 \mathrm{MeV}$ & 2.529 & 2.127 & 10.79 & 1.722 \\
\hline \multirow[t]{5}{*}{ SFHx, $v$-full } & c20p0 & 2.531 & 2.139 & 11.18 & 1.705 \\
\hline & c30p0 & 2.530 & 2.150 & 11.37 & 1.688 \\
\hline & c30p10 & 2.531 & 2.151 & 12.39 & 1.688 \\
\hline & $\mathrm{c} 30 \mathrm{p} 5$ & 2.531 & 2.150 & 11.72 & 1.688 \\
\hline & $\mathrm{c} 40 \mathrm{p} 0$ & 2.527 & 2.160 & 11.51 & 1.671 \\
\hline
\end{tabular}

Notes. " $v$-less" indicates neutrino-less $\beta$-equilibrium, which we use only for the "cold" configurations. " $v$-full" indicates neutrino-full $\beta$-equilibrium with neutrino pressure. $T(\rho)$ is the temperature parameterization, $M_{\mathrm{b}}^{\max }$ is the maximum baryonic mass, $M_{\mathrm{g}}^{\max }$ is the gravitational mass at the maximum baryonic mass, $R$ is the radius of the $M_{\mathrm{b}}^{\max }$ configuration, and $\rho_{c}$ is the central baryon density at which $M_{\mathrm{b}}^{\max }$ obtains. 


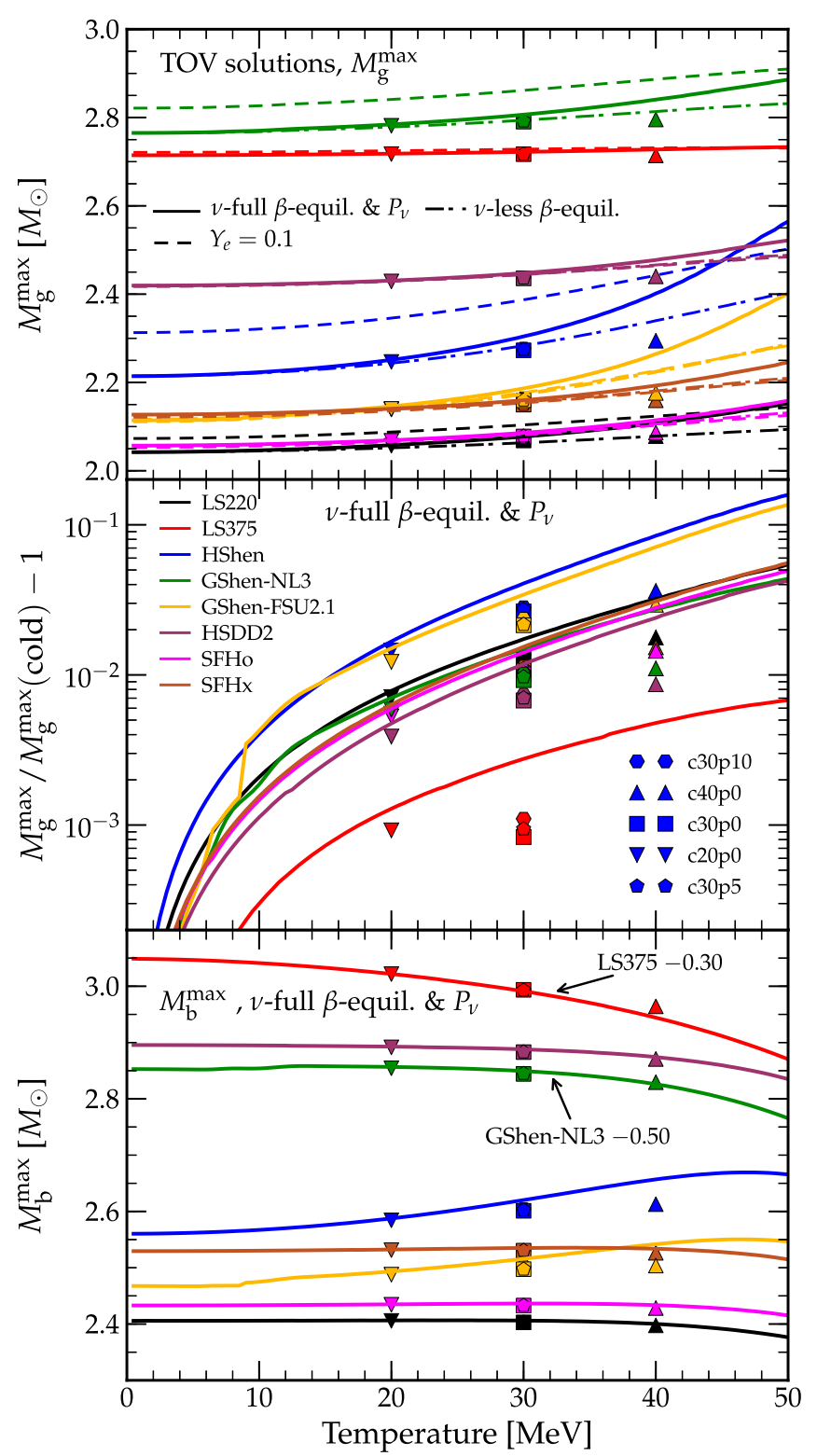

Figure 4. Effect of temperature $T$ on the maximum masses of TOV solutions. Top panel: gravitational mass $M_{\mathrm{g}}^{\max }$ at the maximum baryonic mass for $T=$ const. configurations (lines) and parameterized cXpX profiles (symbols). The $\mathrm{cXpX}$ solutions are computed only for $\nu$-full $\beta$-equilibrium. With increasing $T, M_{\mathrm{g}}^{\max }$ increases. This trend is independent of $Y_{e}$ prescription, but the sensitivity to $Y_{e}$ is highly EOS dependent. Center panel: relative increase of $M_{\mathrm{g}}^{\max }$ with $T$ for solutions in $\nu$-full $\beta$-equilibrium. The increase is modest and below $\sim 10 \%$ even in the $T=$ const. case. Bottom panel: maximum baryonic mass $M_{\mathrm{b}}^{\max }$ that can be supported as a function of temperature. For most EOS, there is little variation in $M_{\mathrm{b}}^{\mathrm{max}}$ at low $T$, but the increasing thermal contribution to the TOV energy density (see Equation (4)) leads to a decrease of $M_{\mathrm{b}}^{\max }$ for high-T solutions. A linear vertical shift of $-0.30(-0.50) M_{\odot}$ has been applied to the LS375 (GShen-NL3) curves to enhance the vertical dynamic range of the plot.

(A color version of this figure is available in the online journal.)

sources curvature (the relativistic gravitational field), the thermal effects lead to a decrease in $M_{\mathrm{b}}^{\max }$ with temperature even if $M_{\mathrm{g}}^{\max }$ is still increasing. The HShen and GShen-FSU2.1 are the only two EOS that exhibit an increase of $M_{\mathrm{b}}^{\max }$ at intermediate to high temperatures, but they too reverse this trend at isothermal $T \gtrsim 50 \mathrm{MeV}$. The LS375 EOS, on the other hand, has monotonically decreasing $M_{\mathrm{b}}^{\max }$ with $T$, which was seen before by O'Connor \& Ott (2011). The more realistic cXpX tempera- ture parameterizations show a similar trend as their isothermal counterparts, but for the HShen and GShen-FSU2.1 EOS, the increase in $M_{\mathrm{b}}^{\max }$ at intermediate $T$ is smaller in these only centrally hot parameterized models.

It is interesting to compare our findings with the results of O'Connor \& Ott (2011), who studied black hole formation through protoNS collapse in failing core-collapse supernovae. These authors found much larger maximum baryonic and gravitational masses of their protoNSs at the onset of collapse than reported here. The collapsing protoNSs in their study have moderately high central temperatures $T \lesssim 40 \mathrm{MeV}$. However, at $\rho \approx 4 \times 10^{14}-10^{15} \mathrm{~g} \mathrm{~cm}^{-3}$, a region of extremely hot material with $T \gtrsim 80-100 \mathrm{MeV}$ is present due to compression of multiple $M_{\odot}$ of accreted shock-heated material. O'Connor \& Ott (2011) demonstrated that this extremely hot region is responsible for the observed thermal enhancement of the maximum protoNS mass. In NSNS mergers the situation is quite different and fully dynamical NSNS merger simulations have not found such extremely hot high-density regions (e.g., Sekiguchi et al. 2011; Oechslin et al. 2007). It is thus unlikely that the findings of O'Connor \& Ott (2011) apply to the merger HMNS case. Table 1 summarizes key parameters of the computed TOV solutions.

\section{RESULTS: AXISYMMETRIC MODELS IN ROTATIONAL EQUILIBRIUM}

\subsection{Uniformly Rotating Configurations}

It has been widely recognized that uniform rotation can support a supramassive NS against gravitational collapse (see, e.g., Friedman et al. 1986; Friedman \& Ipser 1987). A supramassive NS is defined as a stable NS with a mass greater than the maximum mass of a TOV star with the same EOS (CST). At a given central density, the mass that may be supported rises with increasing angular velocity until the material on the NS's equator becomes unbound (the mass-shedding limit). This leads to the supramassive limit, a well defined maximum mass for uniformly rotating NSs with a specified EOS.

In Figure 5, we plot the baryonic mass $M_{\mathrm{b}}$ as a function of maximum baryon density for TOV and uniformly rotating mass-shedding sequences obtained with the LS220 EOS (left panel) and the HShen EOS (right panel). Focusing first on the TOV sequences, one notes that at low central densities $\left(\rho_{\mathrm{b}} \lesssim\right.$ few $\left.\times \rho_{\text {nuc }}\right), M_{\mathrm{b}}$ is significantly increased by thermal effects. This is because the mean density $\bar{\rho}_{\mathrm{b}}$ of such configurations is in the regime in which thermal pressure is of greatest relevance (see Figure 3) and can alter the structure of the bulk of the NS. This carries over to the uniformly rotating case. The extended hot configurations reach mass shedding at lower angular velocities than their cold counterparts, but the extended, low $\bar{\rho}_{\mathrm{b}}$ cores of hot configurations receive sufficient rotational support to yield a higher $M_{\mathrm{b}}$. This, however, is the case only for centrally hot cXp0 configurations. Models with hot envelopes (with parameterizations c30p5 and c30p10) benefit less from rotational support.

With increasing maximum density, the baryonic masses of the TOV models for different temperature parameterizations converge for a given EOS. Near the density at which the maximum mass is reached, the increase in $M_{\mathrm{b}}$ in hot configurations has turned into a slight decrease for models computed with the LS220 EOS and has dropped to $\lesssim 5 \%$ for the HShen EOS (see also Figure 4). The mass-shedding sequences show a more complex behavior with increasing maximum density. As in the TOV 

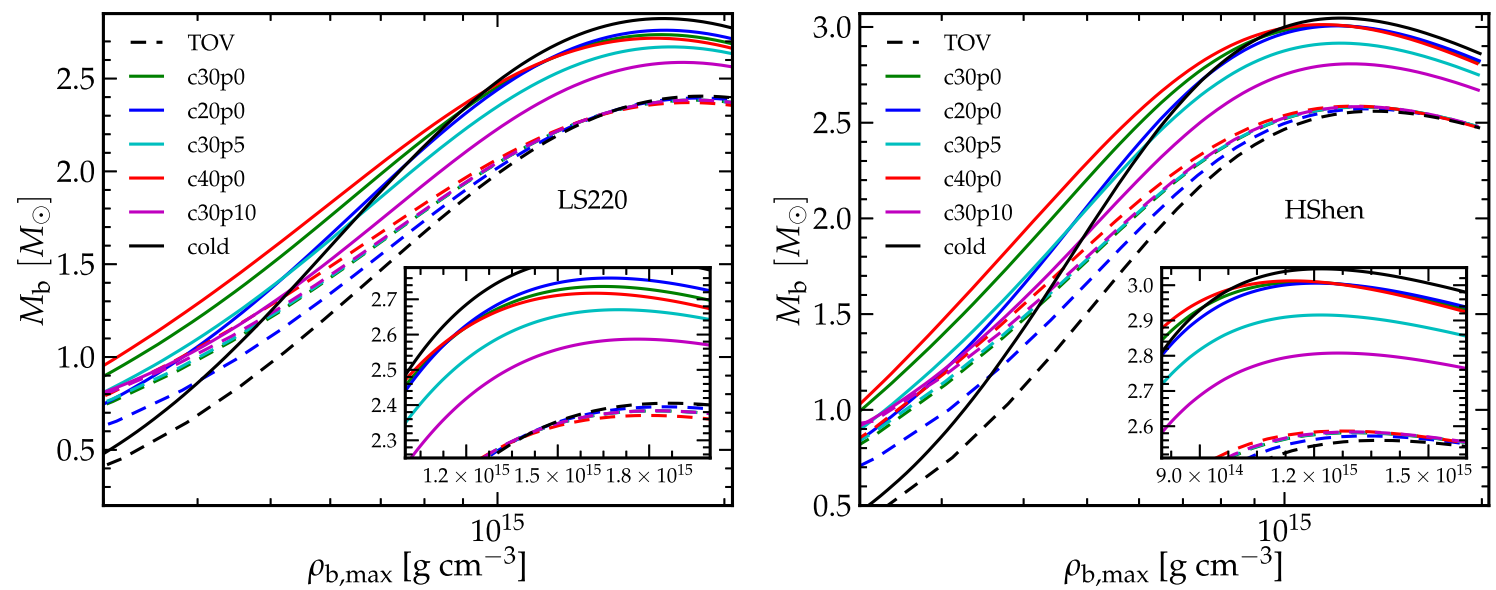

Figure 5. Baryonic mass $M_{\mathrm{b}}$ as a function of maximum baryon density $\rho_{\mathrm{b}, \max }$ of uniformly rotating $(\tilde{A}=0)$ equilibrium models at the mass-shedding limit for different temperature prescriptions (solid lines). We also plot the corresponding TOV sequences (dashed lines) and show results for the LS220 EOS (left panel) and HShen EOS (right panel). There is a large thermal enhancement of $M_{\mathrm{b}}$ at low densities, but the sequences converge toward the cold supramassive limit as the maximum density increases and the configurations become more compact.

(A color version of this figure is available in the online journal.)

Table 2

Uniformly Rotating Neutron Stars at the Supramassive Limit

\begin{tabular}{lcccccr}
\hline \hline Model & $\begin{array}{c}\rho_{\mathrm{b}, \max } \\
\left(10^{15} \mathrm{~g} \mathrm{~cm}^{-3}\right)\end{array}$ & $\begin{array}{c}M_{\mathrm{b}}^{\max } \\
\left(M_{\odot}\right)\end{array}$ & $\begin{array}{c}M_{\mathrm{g}}^{\max } \\
\left(M_{\odot}\right)\end{array}$ & $\begin{array}{c}r_{\mathrm{e}} \\
(\mathrm{km})\end{array}$ & $r_{\mathrm{p} / \mathrm{e}}$ & $\begin{array}{c}\Omega /|W| \\
\left(10^{3} \mathrm{rad} \mathrm{s}^{-1}\right)\end{array}$ \\
\hline LS220 cold & 1.653 & 2.823 & 2.419 & 14.429 & 0.566 & 10.096 \\
LS220 c20p0 & 1.652 & 2.760 & 2.384 & 14.788 & 0.574 & 9.647 \\
LS220 c30p0 & 1.652 & 2.737 & 2.382 & 15.000 & 0.576 & 9.441 \\
LS220 c30p5 & 1.710 & 2.671 & 2.322 & 15.300 & 0.587 & 9.031 \\
LS220 c30p10 & 1.769 & 2.587 & 2.247 & 16.130 & 0.599 & 8.215 \\
LS220 c40p0 & 1.625 & 2.717 & 2.383 & 15.201 & 0.577 & 9.262 \\
\hline & & & & & 0.103 \\
HShen cold & 1.220 & 3.046 & 2.649 & 17.101 & 0.564 & 0.068 \\
HShen c20p0 & 1.196 & 3.006 & 2.629 & 17.760 & 0.573 & 0.101 \\
HShen c30p0 & 1.171 & 3.009 & 2.648 & 18.173 & 0.574 & 7.745 \\
HShen c30p5 & 1.228 & 2.916 & 2.564 & 18.665 & 0.588 & 7.511 \\
HShen c30p10 & 1.261 & 2.808 & 2.467 & 20.070 & 0.604 & 7.086 \\
HShen c40p0 & 1.139 & 3.012 & 2.664 & 18.474 & 0.574 & 0.117 \\
\hline
\end{tabular}

Notes. Summary of mass-shedding uniformly rotating supramassive neutron star configurations at the maximum mass for each EOS and temperature prescription. These models are in $\nu$-less $\beta$-equilibrium (see Section 2.2 ). $\rho_{\mathrm{b}, \max }$ is the central density of the model with the maximum baryonic mass $M_{\mathrm{b}}$. $M_{\mathrm{g}}^{\max }$ is the gravitational mass at the $\rho_{\mathrm{b}, \max }$ at which $M_{\mathrm{b}}^{\max }$ occurs. $r_{\mathrm{e}}$ is the equatorial radius, $r_{\mathrm{p} / \mathrm{e}}$ is the axis ratio, $\Omega$ is the angular velocity, and $T /|W|$ is the ratio of rotating kinetic energy $T$ to gravitational energy $|W|$.

case, the mean density $\bar{\rho}_{b}$ of the NSs increases and less material is experiencing enhanced pressure support due to high temperatures in the cXp0 models. Hence, these models move toward the $M_{\mathrm{b}}^{\max }$ of the cold supramassive limit (see the inset plots in Figure 5). For both EOS, the $M_{\mathrm{b}}^{\max }$ of hot configurations are all lower than the cold value. The cXp0 models reach supramassive limits that are within less than $2 \%$ of the cold supramassive limit for both EOS. The c30p10 and c30p5 models, on the other hand, have $M_{\mathrm{b}}^{\max }$ that are $\sim 5 \%-10 \%$ lower than the cold supramassive limit for both EOS. Table 2 summarizes key parameters of the hot and cold configurations at the supramassive limit.

The systematics of the supramassive limit with temperature prescription becomes clear when considering Figure 6. This figure shows the baryonic mass $M_{\mathrm{b}}$ and gravitational mass $M_{\mathrm{g}}$ for uniformly rotating NSs as a function of angular velocity $\Omega$ for the LS220 and HShen EOS at fixed densities near the maximum of $M_{\mathrm{b}}\left(\rho_{\mathrm{b}, \max }\right)$ (see Table 2). At fixed angular velocity below mass shedding, hotter configurations always yield higher $M_{\mathrm{g}}$ than their colder counterparts. For the LS220 EOS, as in the TOV case discussed in the previous section 3, hotter configurations have lower $M_{\mathrm{b}}$. In the case of the HShen EOS, which generally yields less compact equilibrium models, the opposite is true, but the increase in $M_{\mathrm{b}}$ caused by thermal support is smaller than the increase in $M_{\mathrm{g}}$.

With increasing $\Omega$, the mass-shedding limit is approached and hotter configurations systematically reach the mass shedding limit at lower angular velocities. The reason for this is best illustrated by comparing c30p0 models with c30p10 and c30p5 models, which have a high-temperature plateau at low densities of $10 \mathrm{MeV}$ and $5 \mathrm{MeV}$, respectively. At low angular velocities, all $\mathrm{c} 30 \mathrm{pX}$ models show the same thermal increase in $M_{\mathrm{g}}$. However, the high pressure at low densities in the c30p10 and c30p5 models leads to significantly larger radii compared to the model without temperature plateau. Consequently, as $\Omega$ is increased, the configurations with plateau reach the massshedding limit at lower $\Omega$. For the LS220 EOS, the c30p10 

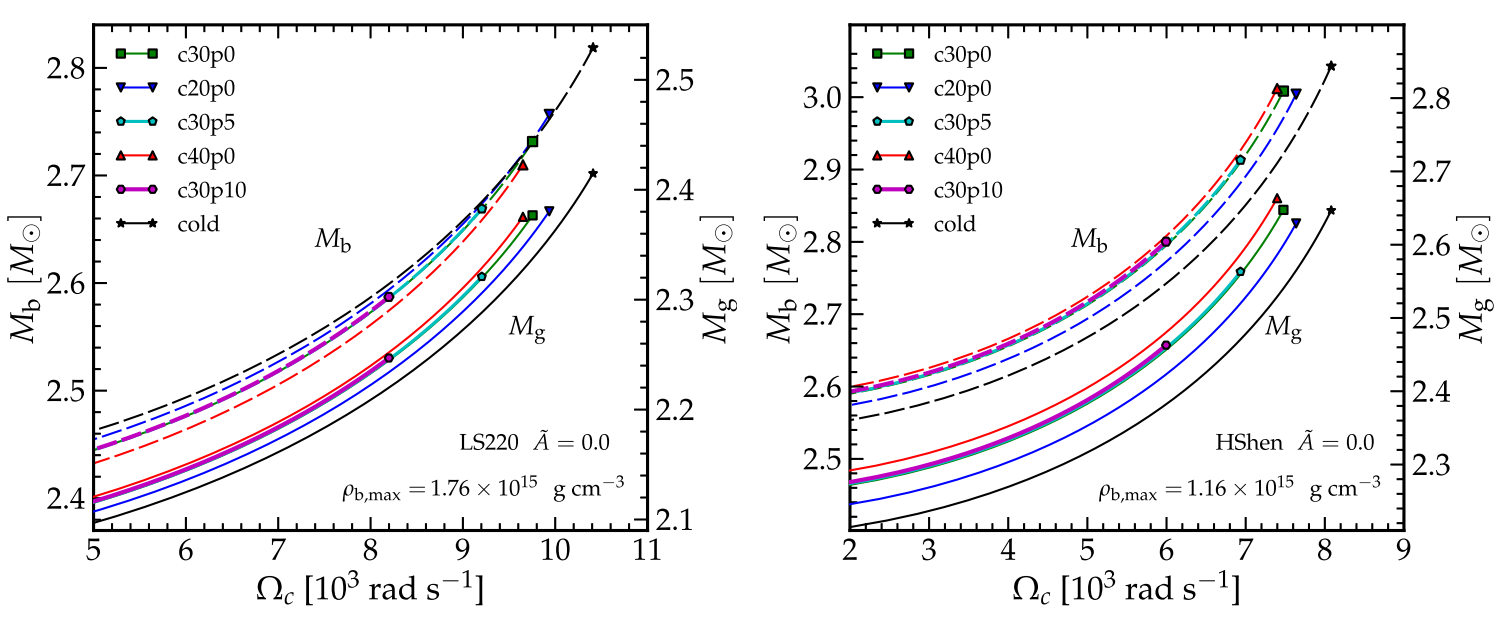

Figure 6. Gravitational mass $\left(M_{\mathrm{g}}\right.$, solid lines, right ordinate) and baryonic mass ( $M_{\mathrm{b}}$, dashed lines, left ordinate) as a function of angular velocity $\Omega$ for uniformly spinning models at a fixed density near the density that yields the maximum $M_{\mathrm{b}}$ for the LS220 (left panel) and the HShen EOS (right panel). The sequences terminate at the mass-shedding limit, which is the point with the maximum angular velocity for a specific temperature prescription. Configurations with higher temperatures, in particular, the c30p5 and c30p10 models with high-temperature plateaus at low densities, have larger radii than colder models and thus reach the mass-shedding limit at lower angular velocities. Hence, such models have lower maximum masses at the supramassive limit than colder models. Note that hotter models with the LS220 have lower baryonic masses than colder models.

(A color version of this figure is available in the online journal.)

Table 3

Differentially Rotating Hypermassive Neutron Stars

\begin{tabular}{|c|c|c|c|c|c|c|c|c|}
\hline Model & $\begin{array}{c}\rho_{\mathrm{b}, \max } \\
\left(10^{15} \mathrm{~g} \mathrm{~cm}^{-3}\right)\end{array}$ & $\begin{array}{l}M_{\mathrm{b}}^{\max } \\
\left(M_{\odot}\right)\end{array}$ & $\begin{array}{l}M_{\mathrm{g}}^{\max } \\
\left(M_{\odot}\right)\end{array}$ & $\begin{array}{c}r_{\mathrm{e}} \\
(\mathrm{km})\end{array}$ & $r_{\mathrm{p} / \mathrm{e}}$ & $\tilde{A}$ & $\begin{array}{c}\Omega_{c} \\
\left(10^{3} \mathrm{rad} \mathrm{s}^{-1}\right)\end{array}$ & $T /|W|$ \\
\hline LS220 cold & 0.993 & 3.648 & 3.140 & 17.258 & 0.376 & 0.5 & 15.476 & 0.244 \\
\hline LS220 c20p0 & 0.852 & 3.573 & 3.124 & 18.538 & 0.364 & 0.6 & 15.047 & 0.243 \\
\hline LS220 c 30 p0 & 0.706 & 3.568 & 3.167 & 19.611 & 0.344 & 0.7 & 14.888 & 0.249 \\
\hline LS220 c30p5 & 0.600 & 3.413 & 3.064 & 21.870 & 0.320 & 0.9 & 14.461 & 0.250 \\
\hline LS 220 c 30 p 10 & 0.990 & 3.090 & 2.723 & 19.208 & 0.421 & 0.9 & 16.330 & 0.187 \\
\hline LS220 c40p0 & 0.692 & 3.597 & 3.211 & 19.931 & 0.344 & 0.7 & 14.677 & 0.249 \\
\hline HShen cold & 0.766 & 4.101 & 3.562 & 19.800 & 0.372 & 0.5 & 13.450 & 0.245 \\
\hline HShen $\mathrm{c} 20 \mathrm{p} 0$ & 0.641 & 4.076 & 3.585 & 21.352 & 0.360 & 0.6 & 13.042 & 0.245 \\
\hline HShen c $30 \mathrm{p} 0$ & 0.532 & 4.099 & 3.650 & 22.305 & 0.344 & 0.7 & 13.131 & 0.249 \\
\hline HShen c30p5 & 0.517 & 3.942 & 3.527 & 24.371 & 0.340 & 0.8 & 12.426 & 0.243 \\
\hline HShen c30p10 & 0.646 & 3.529 & 3.141 & 23.521 & 0.400 & 1.0 & 13.934 & 0.196 \\
\hline HShen c40p0 & 0.514 & 4.148 & 3.708 & 22.701 & 0.344 & 0.7 & 12.888 & 0.249 \\
\hline
\end{tabular}

Notes. Summary of the differentially rotating HMNS configurations with the largest baryonic masses for each EOS and temperature prescription. These configurations are obtained in a sequence from $\tilde{A}=0$ to $\tilde{A}=1$ with spacing $\delta \tilde{A}=0.1$ and are to be seen as lower bounds on the maximum achievable masses. The sequences considered here exclude dynamically nonaxisymmetrically unstable models with ratio of rotational kinetic energy to gravitational energy $T /|W|>0.25$. The quantities listed in the table are the following: $\rho_{\mathrm{b}, \max }$ is the baryon density at which the maximum baryonic mass $M_{\mathrm{b}}^{\max }$ occurs, $M_{\mathrm{g}}^{\max }$ is the gravitational mass at that density, $r_{\mathrm{e}}$ is the equatorial radius of the configuration, $r_{\mathrm{p} / \mathrm{e}}$ is its axis ratio, $\tilde{A}$ is the differential rotation parameter at which $M_{\mathrm{b}}^{\max }$ obtains. $\Omega_{c}$ is the central angular velocity of the configuration and $T /|W|$ is its ratio of rotational kinetic energy to gravitational energy. We note that the accuracy of the results listed in this table is set by the step size in $r_{\mathrm{p} / \mathrm{e}}$, which we set to $\delta r_{\mathrm{p} / \mathrm{e}}=0.004$.

sequence terminates at $\sim 8200 \mathrm{rad} \mathrm{s}^{-1}$, the c30p5 sequence terminates at $\sim 9200 \mathrm{rad} \mathrm{s}^{-1}$, while the $\mathrm{c} 30 \mathrm{p} 0$ sequence does not terminate before $\sim 9800 \mathrm{rad} \mathrm{s}^{-1}$. The HShen model sequences show the same qualitative trends.

\subsection{Differentially Rotating Configurations}

Differential rotation can provide centrifugal support at small radii while allowing a NS configuration to stay below the massshedding limit at its equatorial surface. Differentially rotating equilibrium configurations have been shown to support masses well in excess of the supramassive limit (e.g., Ostriker et al. 1966; Baumgarte et al. 2000; Morrison et al. 2004). Such configurations are referred to as "hypermassive." However, since there is (mathematically speaking) an infinite number of possible differential rotation laws, it is impossible to define a formal "hypermassive limit" for the maximum mass of HMNSs in the way it is possible for uniformly rotating supramassive NSs. Nevertheless, we can study the systematics of the supported baryonic (and gravitational) masses with variations in the HMNS temperature profile, maximum baryon density, and degree and rate of differential rotation for the rotation law considered in this study, which is not drastically different from what is found in merger simulations (e.g., Shibata et al. 2005).

In Figure 7, we show the supported baryonic mass $M_{\mathrm{b}}$ as a function of maximum baryon density $\rho_{\mathrm{b}, \max }$ for cold, c20p 0 , and $\mathrm{c} 40 \mathrm{p} 0$ temperature prescriptions, both EOS, and for different choices of $\tilde{A}$ (see Table 3 for a summary of quantitative results). The curves represent configurations with the minimum $r_{\mathrm{p} / \mathrm{e}}$ 

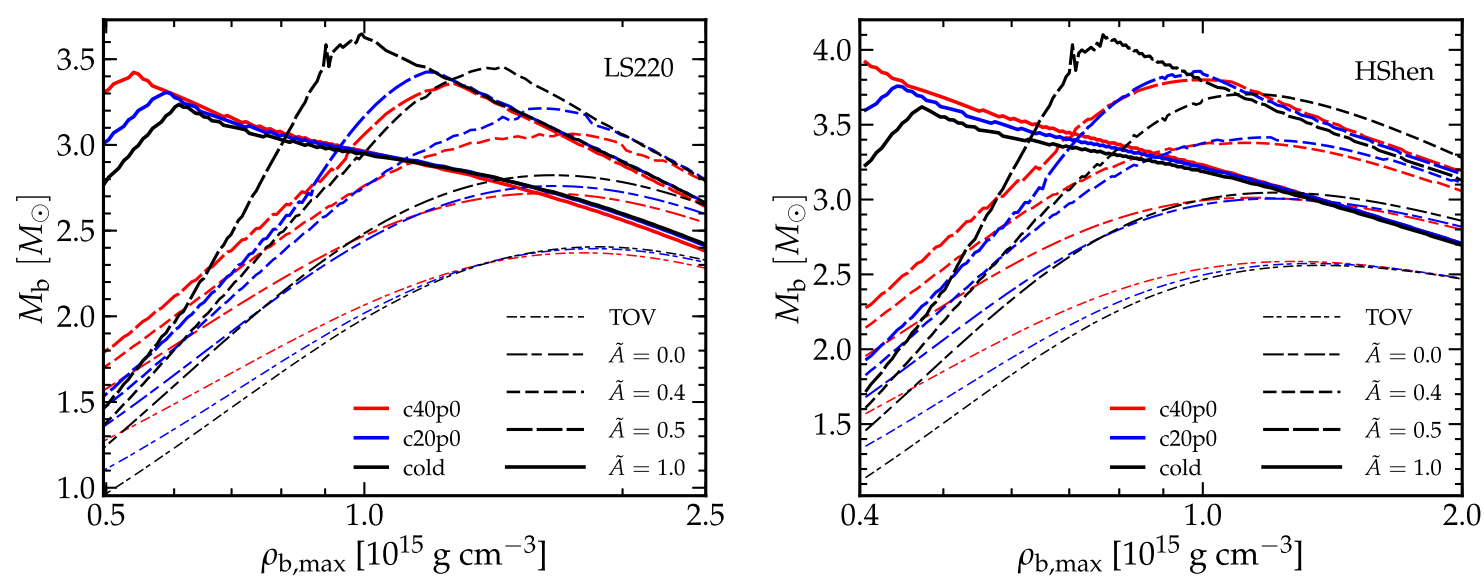

Figure 7. Maximum baryonic mass configurations for sequences of uniformly rotating $(\tilde{A}=0)$ and differentially rotating $(\tilde{A}=\{0.4,0.5,1.0\})$ models with cold, $\mathrm{c} 20 \mathrm{p} 0$, and c40p0 temperature parameterizations and the LS220 EOS (left panel) and HShen EOS (right panel). We note that for differentially rotating models these curves represent lower limits on the maximum baryonic mass (i.e., the solver fails to converge at lower axis ratios without reaching the true mass shedding limit). We limit the sequences to models with $T /|W| \lesssim 0.25$ and this limit defines the rising part of the graphs for $\tilde{A}=1$ at low densities. We show the TOV case (thinnest and shortest dash-dotted lines) for comparison. The raggedness of the curves with $\tilde{A} \gtrsim 0.4$ is a consequence of finite resolution in the parameter $r_{\mathrm{p} / \mathrm{e}}$ that is varied to find the maximum mass at a given $\rho_{\mathrm{b}, \max }$. Thermal effects are most pronounced at low densities and for high $\tilde{A}$. For uniform and moderate differential rotation, hotter models have lower global maximum $M_{\mathrm{b}}$ than colder models.

(A color version of this figure is available in the online journal.)

at which an equilibrium solution is found by the CST solver (i.e., the most rapidly spinning setup). Note that the peaks of these curves represent only lower limits on the maximum HMNS mass. In addition, we plot only solutions with ratios $T /|W|$ of rotational kinetic energy $T$ to gravitational energy $|W|$ below $25 \%$, since more rapidly spinning models would be dynamically nonaxisymmetrically unstable (Chandrasekhar 1969; Baiotti et al. 2007). It is this limit which defines the rising branch of the $M_{\mathrm{b}}\left(\rho_{\mathrm{b}, \max }\right)$ curve at the lowest densities in Figure 7 for $\tilde{A}=1.0$. Note that many of these configurations may still be unstable to secular rotational instabilities or rotational shear instabilities (e.g., Watts et al. 2005; Ott et al. 2007; Corvino et al. 2010).

The overall shape of the $M_{\mathrm{b}}\left(\rho_{\mathrm{b}, \max }\right)$ curves in Figure 7 is qualitatively similar to what is shown in Figure 1 of Baumgarte et al. (2000) for $\Gamma=2$ polytropes and Figure 2 of Morrison et al. (2004) for the cold Friedman-Pandharipande EOS (Friedman \& Pandharipande 1981). The LS220 and HShen EOS yield qualitatively very similar results, but the supported HMNS masses found by the CST solver are, as expected, systematically higher for models with the HShen EOS than for those using the LS220 EOS. One notes, however, interesting variations with temperature prescription. At low $\rho_{\mathrm{b}, \max }$, thermal pressure support leads to increased $M_{\mathrm{b}}$ and more differentially rotating configurations have higher $M_{\mathrm{b}}$. Sequences with $\tilde{A} \lesssim 0.5$ show similar systematics with density and temperature prescription as the uniformly spinning ones discussed in Section 4.1: As the density increases, hot configurations converge toward the cold sequence and reach their maximum $M_{\mathrm{b}}$ near and below the maximum of the cold sequence. Models with $\tilde{A} \gtrsim 0.5$, on the other hand, have more steeply rising curves with $\rho_{\mathrm{b}, \max }$ and are discontinuous (i.e., exhibit a "kink") at their global maxima. At these points quasitoroidal solutions appear. Furthermore, the slope of the curve describing (as a function of $\rho_{\mathrm{b}, \max }$ ) the axis ratios $r_{\mathrm{p} / \mathrm{e}}$ at which the solver stops converging discontinuously changes sign. We attribute this behavior, which was also observed by Morrison et al. 2004, to a bifurcation of the sequence between models, which continue shrinking in axis ratio until they become completely toroidal $\left(r_{\mathrm{p} / \mathrm{e}}=0\right)$, and less extreme models that stay quasitoroidal or spheroidal. Beyond the "kink" in $\tilde{A} \gtrsim 0.5$ sequences, thermal effects play little role.

The lower bounds of the range of $\rho_{\mathrm{b}, \max }$ shown in the two panels of Figure 7 (and also Figure 8) are chosen for the following reason: fully dynamical merger simulations by, e.g., Sekiguchi et al. (2011), Baiotti et al. (2008), Shibata et al. (2005), Kiuchi et al. (2009), Bauswein et al. (2012), and Thierfelder et al. (2011), all suggest a rule of thumb that the postmerger maximum baryon density of the HMNS is typically not less than $\sim 80 \%$ of the central density of the progenitor NSs. We can derive a rather solid EOS-dependent lower limit on $\rho_{\mathrm{b}, \max }$ for HMNS remnants from (equal mass) NSNS mergers in the following way. In order to form an HMNS, constituent equal-mass NSs must at the very least have a mass that is $50 \%$ of the maximum mass in the cold TOV limit. Hence, the premerger central density must at least be that of a TOV solution with $M_{\mathrm{b}}=0.5 M_{\mathrm{b}}^{\text {max,TOV }}$. Using the aforementioned empirical result from merger simulations, we arrive at

$$
\rho_{\mathrm{b}, \min }=0.8 \rho_{\mathrm{b}, \mathrm{TOV}}\left(M_{\mathrm{b}}=M_{\mathrm{b}, \max } / 2\right) .
$$

For the LS220 EOS, $\rho_{\mathrm{b}, \operatorname{TOV}}\left(M_{\mathrm{b}}=M_{\mathrm{b}, \max } / 2\right) \sim 5.8 \times$ $10^{14} \mathrm{~g} \mathrm{~cm}^{-3}$ and occurs at $M_{\mathrm{b}}\left(M_{\mathrm{g}}\right)$ of $1.19 M_{\odot}\left(1.10 M_{\odot}\right)$. For the HShen EOS, $\rho_{\mathrm{b}, \mathrm{TOV}}\left(M_{\mathrm{b}}=M_{\mathrm{b}, \max } / 2\right) \sim 4.4 \times 10^{14} \mathrm{~g} \mathrm{~cm}^{-3}$ and occurs at $M_{\mathrm{b}}\left(M_{\mathrm{g}}\right)$ of $1.28 M_{\odot}\left(1.20 M_{\odot}\right)$. Applying the density cut given by Equation (7) excludes most dynamically nonaxisymmetrically unstable configurations.

Figure 8, like Figure 7, shows baryonic mass as a function of maximum baryon density for both EOS and a variety of $\tilde{A}$, but contrasts models c30p5 and c30p10, which have hot plateaus at low densities, with cold models. The qualitative features discussed in the following are identical for both EOS. In the TOV case and at low densities, $M_{\mathrm{b}}$ is enhanced primarily by the hot core, since nonrotating solutions are compact and dominated by $\rho_{\mathrm{b}} \gtrsim 10^{14} \mathrm{~g} \mathrm{~cm}^{-3}$, where the high-temperature plateaus play no role. At higher densities, the $M_{\mathrm{b}}$ curves of hot models converge to near or below the cold TOV maximum $M_{\mathrm{b}}$. The situation 

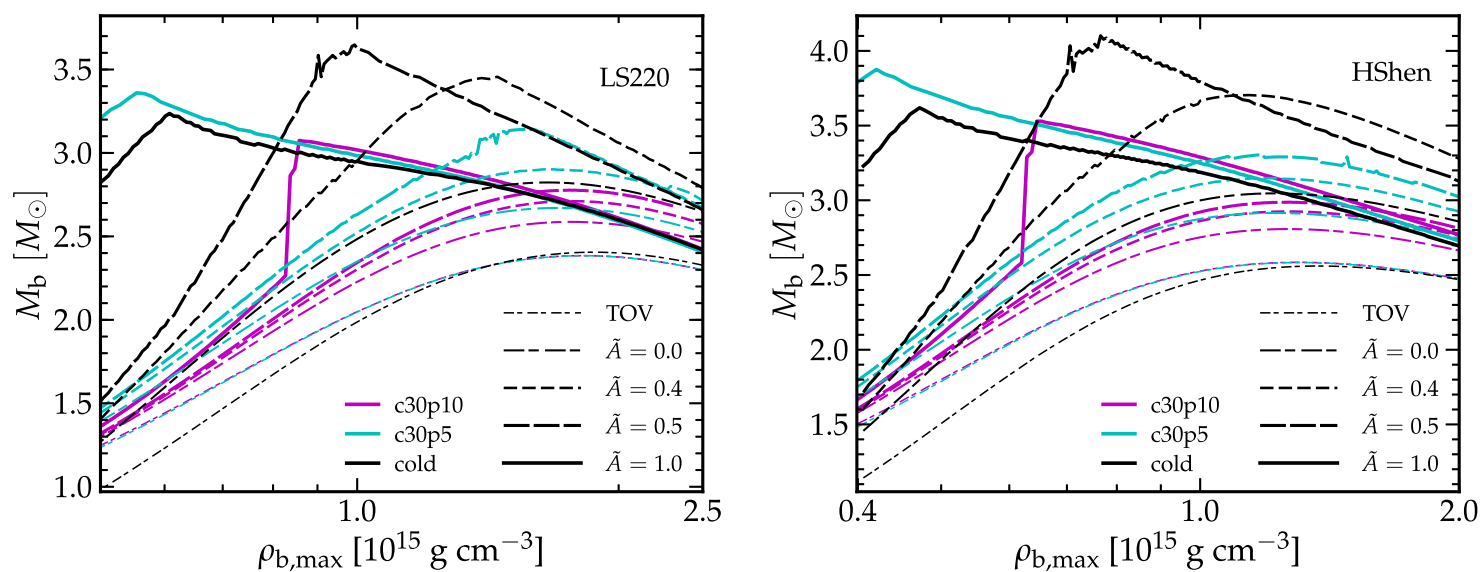

Figure 8. Same as Figure 7, but comparing cold configurations with models with the c30p5 and c30p10 temperature prescriptions, which have a hot plateau at low densities. The overall systematics are the same for the LS220 EOS (left panel) and the HShen EOS (right panel). In the TOV case, $M_{\mathrm{b}}$ is thermally enhanced at low densities, but the global maximum of $M_{\mathrm{b}}$ of hot configurations is near that of the cold TOV solution. Uniformly and moderately differentially rotating sequences of c30p10 and c30p5 models have systematically smaller maximum masses than cold models throughout the considered density range. Only very differentially rotating models $(\tilde{A} \gtrsim 0.7 ; \tilde{A}=1.0$ shown here) exhibit a thermal enhancement of the maximum mass at low to intermediate densities. The c30p10 sequence for $\tilde{A}=1.0$ exhibits a discontinuous jump, which occurs when the sequence transitions from spheroidal to quasitoroidal shape. See text for discussion.

(A color version of this figure is available in the online journal.)

is different for uniformly and moderately differentially rotating models $(\tilde{A} \lesssim 0.5)$. Rotation shifts these configurations to lower mean densities and the hot plateaus lead to equatorially bloated solutions. These reach their minimum $r_{\mathrm{p} / \mathrm{e}}$ for which a solution can be found at lower angular velocities. Hence, centrifugal support is weaker and the configuration with the hottest plateau has the lowest $M_{\mathrm{b}, \max }$. The behavior is different at high degrees of differential rotation $(\tilde{A}=1)$. The cold and the c30p5 models are HMNSs and quasitoroidal already at the lowest densities shown in Figure 8. The c30p5 sequence has slightly larger $M_{\mathrm{b}}$ than the cold sequence. The c30p10 sequence, however, is spheroidal at low $\rho_{\mathrm{b}, \max }$ and then discontinuously transitions to the quasitoroidal branch, which is marked by a large jump in $M_{\mathrm{b}}$.

In order to illustrate this discontinuous behavior further, we plot in Figure 9 the equatorial radius of equilibrium solutions as a function of central angular velocity at $\tilde{A}=1$ and for three different fixed $\rho_{\mathrm{b}, \max }$. We show curves obtained with the LS220 EOS for the cold, c30p5, and c30p10 temperature prescriptions. The curves are parameterized by decreasing $r_{\mathrm{p} / \mathrm{e}}$ and terminate at the smallest value at which the solver converges. The three densities are chosen so that the first two are below and the third is above the jump of the c30p10 curve in Figure 8 . At all $\rho_{\mathrm{b}, \max }$, the hot configurations have significantly larger radii than the cold models, but decreasing $r_{\mathrm{p} / \mathrm{e}}$ leads to increasing $\Omega_{c}$ and only modest radius changes for cold and c30p5 models. This is very different for the c30p10 sequence. At $\rho_{\mathrm{b}, \max }=7.11 \times 10^{14} \mathrm{~g} \mathrm{~cm}^{-3}$ these models do not become quasitoroidal and the $r_{\mathrm{e}}-\Omega_{c}$ mapping becomes double-valued as the decrease in $r_{\mathrm{p} / \mathrm{e}}$ turns from a decrease of $r_{\mathrm{p}}$ at nearly fixed $r_{\mathrm{e}}$ and increasing $\Omega_{c}$ into a steep increase of $r_{\mathrm{e}}$ and a decrease of $\Omega_{c}$. As $\rho_{\mathrm{b}, \max }$ increases, less material is at low densities where thermal pressure support is strong in the c30p10 models. Consequently, the solutions are more compact and stay so to smaller $r_{\mathrm{p} / \mathrm{e}} \cdot \rho_{\mathrm{b}, \max }=8.16 \times 10^{14} \mathrm{~g} \mathrm{~cm}^{-3}$ is the critical density at which the very last point in the sequence of decreasing $r_{\mathrm{p} / \mathrm{e}}$ (the one shown in Figure 8) jumps discontinuously to large $r_{\mathrm{e}}$. At $\rho_{\mathrm{b}, \max }=9.21 \times 10^{14} \mathrm{~g} \mathrm{~cm}^{-3}$, which is above the critical density for c30p10 in Figure 8, the c30p10 models become

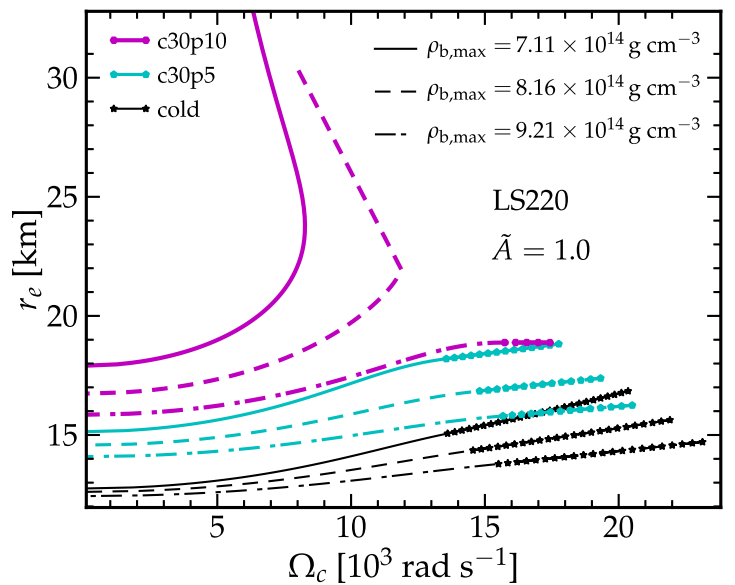

Figure 9. Equatorial radii $r_{\mathrm{e}}$ vs. central angular velocity $\Omega_{c}$ in sequences parameterized by the axis ratio $r_{\mathrm{p} / \mathrm{e}}$ for models using the LS220 EOS, differential rotation parameter $\tilde{A}=1.0$, and cold, c30p5, and c30p10 temperature parameterizations. We show curves for three densities, two below the discontinuous jump of the c 30 p 10 curve in Figure 8 and one above. At the same density, hotter configurations have larger radii and transition to quasitoroidal shape (marked by dots) at higher $\Omega_{c}$. The transition between spheroidal and quasitoroidal shape is discontinuous in $\rho_{\mathrm{b}, \max }$ for critical models at the minimum $r_{\mathrm{p} / \mathrm{e}}$ that can be found (shown in Figures 7 and 8), but smooth in $r_{\mathrm{p} / \mathrm{e}}$ at fixed $\rho_{\mathrm{b}, \max }$. The lowdensity sequences with the $\mathrm{c} 30 \mathrm{p} 10$ temperature prescription $(10 \mathrm{MeV}$ plateau at low densities; see Section 2.2) become double valued in $\Omega_{c}$ with increasing $r_{\mathrm{p} / \mathrm{e}}$, stay spheroidal and have very large $r_{\mathrm{e}}$.

(A color version of this figure is available in the online journal.)

quasitoroidal as $r_{\mathrm{p} / \mathrm{e}}$ decreases and $\Omega_{c}$ increases. They exhibit the same systematics as the c30p5 and cold models. We note that what we have described for the c30p10 models also occurs for the c30p5 models, although at significantly lower densities $\rho_{\mathrm{b}, \max } \lesssim 5 \times 10^{14} \mathrm{~g} \mathrm{~cm}^{-3}$ and even the cold models show similar trends at low densities.

The sequences shown in Figures 7 and 8 are extreme configurations in the sense that models with smaller $r_{\mathrm{p} / \mathrm{e}}$ cannot be found by the CST solver and may not exist for the rotation law that we consider here. Real HMNS may not by such critical rotators. In Figure 10, we plot $M_{\mathrm{b}}$ and $M_{\mathrm{g}}$ for the 


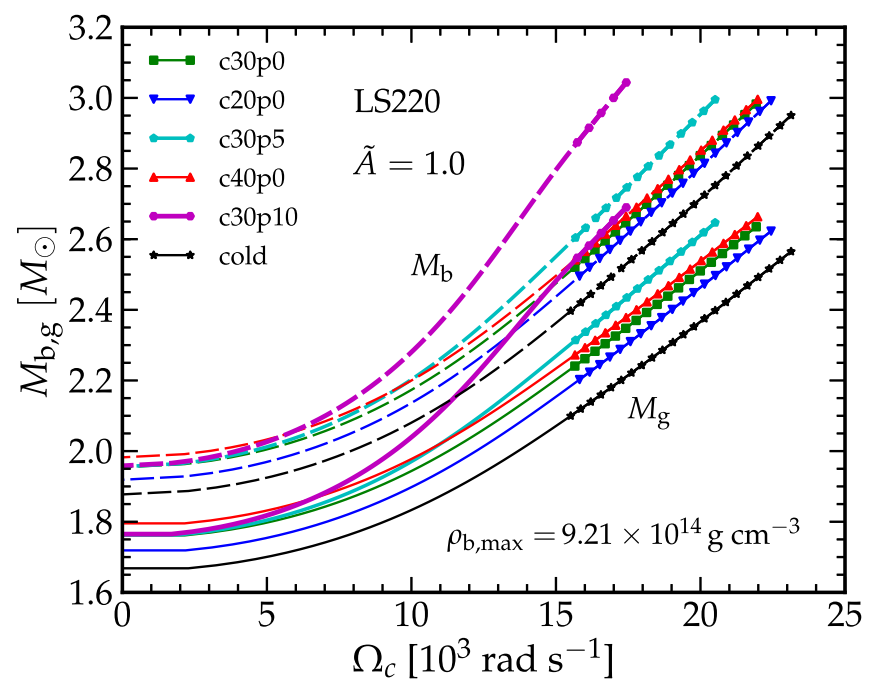

Figure 10. Baryonic mass $M_{\mathrm{b}}$ and gravitational mass $M_{\mathrm{g}}$ vs. central angular velocity $\Omega_{c}$ parameterized by the axis ratio $r_{\mathrm{p} / \mathrm{e}}$ at fixed degree of differential rotation $\tilde{A}=1$, and fixed maximum density of $\rho_{\mathrm{b}, \max }=9.21 \times 10^{14} \mathrm{~g} \mathrm{~cm}^{-3}$. Curves for all temperature parameterizations are shown for the LS220 EOS. Quasitoroidal configurations are marked by symbols and the transitions between spheroidal and quasitoroidal solutions are smooth. The end points of all graphs correspond to the values plotted in Figures 7 and 8 for the various temperature prescriptions at $\tilde{A}=1.0$ and the $\rho_{\mathrm{b}, \max }$ chosen here. Sequences with hot plateaus (using temperature prescriptions c30p5 and c30p10) exhibit significant thermal enhancements of $M_{\mathrm{b}}$ and $M_{\mathrm{g}}$ at rapid rotation rates, but have lower maximum rotation rates due to their larger radii.

(A color version of this figure is available in the online journal.)

LS220 EOS as a function of central angular velocity $\Omega_{c}$ and temperature prescription. We fix the degree of differential rotation to $\tilde{A}=1$ and show sequences in $\Omega_{c}$ for a fixed maximum density $\rho_{\mathrm{b}, \max }=9.21 \times 10^{14} \mathrm{~g} \mathrm{~cm}^{-3}$, which is the highest density shown in Figure 9. The transition to quasitoroidal shape is smooth and quasitoroidal configurations are marked with symbols. The end points of the $M_{\mathrm{b}}$ curves shown in Figure 10 and in Figure 9 correspond to the $M_{\mathrm{b}}$ values of the $\tilde{A}=1$ curves in Figures 7 and 8 at $9.21 \times 10^{14} \mathrm{~g} \mathrm{~cm}^{-3}$.

Figure 10 shows that, as in the case of uniform rotation (see Figure 6), hotter subcritically differentially spinning configurations have higher $M_{\mathrm{g}}$. At the density chosen for this plot, they also have higher $M_{\mathrm{b}}$, but at the higher densities at which the masses of uniformly spinning models peak, the $M_{\mathrm{b}}$ of hotter configurations are smaller than those of colder ones. It is particularly remarkable that the models with the hot plateau at low densities show the greatest thermal enhancement. They also transition to a quasitoroidal shape last but terminate the earliest in $\Omega_{c}$. Nevertheless, for the $\rho_{\mathrm{b}, \max }$ chosen here, they can support slightly more mass at critical rotation than their counterparts without low-density temperature plateau.

\section{DISCUSSION AND COMPARISON WITH THREE-DIMENSIONAL NSNS SIMULATIONS}

\subsection{The Stability of HMNS Equilibrium Sequences}

The existence of a maximum mass for equilibrium sequences of nonrotating (TOV) NSs is one of the most important astrophysical consequences of general relativity and, hence, is well known in the study of compact objects. The parameter space of hot differentially rotating HMNS models studied here is vast and complex. In the following, we briefly review the classical results on the stability of stationary NSs and formulate how one may reason regarding the stability of HMNS equilibrium models.

A particular useful approach to the stability problem is the turning-point method of Sorkin (1982). The turning-point method allows one to reason about the stability of sequences of equilibrium solutions solely by examining the parameter space of equilibrium models without dynamical simulations or linear perturbation analysis. The turning-point method has been used extensively in previous work on the stability of cold and uniformly rotating NSs (e.g., CST; Friedman et al. 1988; Stergioulas \& Friedman 1995; Read et al. 2009).

An equilibrium sequence is a one-dimensional slice from the space of equilibrium models indexed by some parameter. Here we use $\rho_{\mathrm{b}, \max }$ as our sequence parameter. A model in the space of equilibrium models may be defined by the following conserved quantities: the gravitational mass $M_{\mathrm{g}}$, baryonic mass $M_{\mathrm{b}}$, total angular momentum $J$, and total entropy $S$. Generally, as one changes the sequence parameter, $\rho_{\mathrm{b}, \max }$, the quantities $\left(M_{\mathrm{g}}, M_{\mathrm{b}}\right.$, $J, S$ ) will vary. A turning point in the sequence occurs when 3 out of 4 of the derivatives $d / d \rho_{\mathrm{b}, \max }$ of $\left(M_{\mathrm{g}}, M_{\mathrm{b}}, J, S\right)$ vanish. For this point in $\rho_{\mathrm{b}, \max }$, the turning point theorem shows (1) that the derivative of the fourth quantity in the tuple also vanishes, and (2) that the sequence must have transitioned from stable to unstable (Sorkin 1982; Kaplan 2014). This characterization of the space of equilibrium models relies on the assumption that the change in $M_{\mathrm{g}}$ depends to first order only on the total changes in baryonic mass $M_{\mathrm{b}}$, angular momentum $J$, and entropy $S$, and not on changes to their higher moments. That is, changes in the distribution of entropy, baryonic mass and angular momentum. In nature, this will generally not be the case, since cooling and angular momentum redistribution will change the entropy and angular momentum distributions, respectively. However, these changes will be slow and not drastic so that changes to the total energy due to changes in these higher order moments will be small. We account for such changes approximately by considering different degrees of differential rotation and a range of temperature prescriptions in the following.

If we are considering the special case of zero-temperature configurations, then the entropy $S$ is no longer relevant to the equilibrium's stability, since the change to the configuration's energy due to a change in entropy is also zero. In this case, a turning point may be identified when two out of three of the set $d / d \rho_{\mathrm{b}, \max }\left(M_{\mathrm{g}}, M_{\mathrm{b}}, J\right)$ are zero. Zero temperature is a very good approximation for our cold equilibrium models. In Figure 11, we plot $M_{\mathrm{g}}$ along constant $M_{\mathrm{b}}$ sequences with $M_{\mathrm{b}}=2.9 M_{\odot}$ for the HShen EOS $\left(M_{\mathrm{b}}=2.9 M_{\odot}\right.$ corresponds to $M_{\mathrm{b}}$ of an HMNS formed from two NSs of $M_{\mathrm{g}}=1.35 M_{\odot}$, assuming no mass loss). All of these curves have a minimum located at $\rho_{\mathrm{b}, \max } \gtrsim 1 \times 10^{15} \mathrm{~g} \mathrm{~cm}^{-3}$.

For the cold sequences, these minima are turning points because $d M_{\mathrm{g}} / d \rho_{\mathrm{b}, \max }$ and $d M_{\mathrm{b}} / d \rho_{\mathrm{b}, \max }$ are both zero. Any models along those curves at densities in excess of $\rho_{\mathrm{b}, \max }$ at the minima are secularly unstable to collapse. For the hot temperature parameterizations, ${ }^{10}$ the minima are only approximations to the turning point (which we shall call approximate turning points) because only two out of four $\left(d M_{\mathrm{g}} / d \rho_{\mathrm{b}, \max }\right.$ and $\left.d M_{\mathrm{b}} / d \rho_{\mathrm{b}, \max }\right)$ of the derivatives of $\left(M_{\mathrm{g}}, M_{\mathrm{b}}, J, S\right)$ are zero. We argue that these approximate turning points are good indicators of the

\footnotetext{
10 We show only the $\mathrm{c} 40 \mathrm{p} 0$ and cold temperature parameterizations in Figure 11, because we find them to be the limiting cases. All other parameterizations have minima at intermediate locations in the $\left(M_{\mathrm{g}}, \rho_{\mathrm{b}, \max }\right)$ plane.
} 


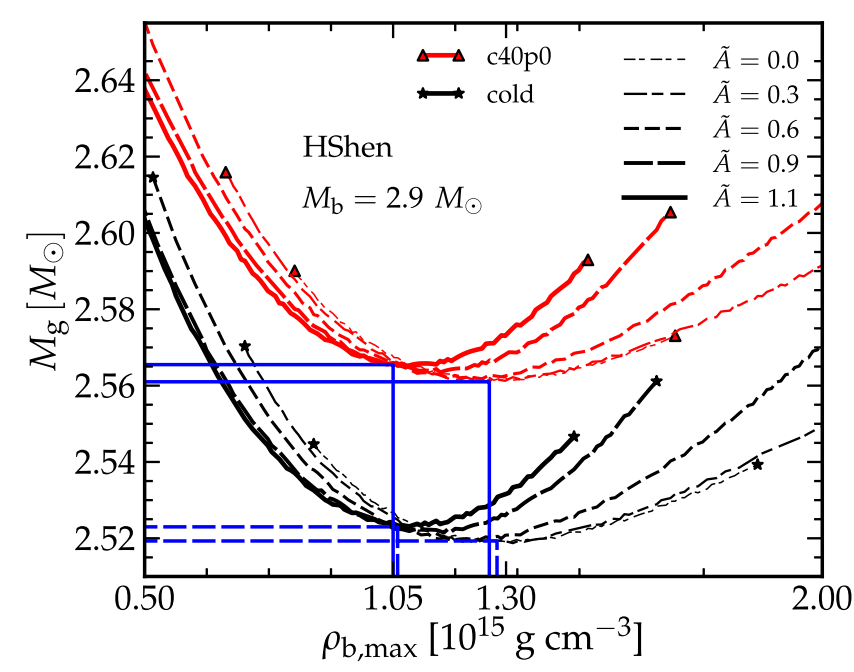

Figure 11. Gravitational mass $M_{\mathrm{g}}$ as a function of maximum baryon density $\rho_{\mathrm{b}, \max }$ for models with $M_{\mathrm{b}}=2.9 M_{\odot}$. Each curve is for a fixed degree of differential rotation $\tilde{A}$, with the axis ratios $r_{\mathrm{p} / \mathrm{e}}$ chosen such that $M_{\mathrm{b}}=2.9 M_{\odot}$. Symbols mark equilibrium solutions at the minimum $r_{\mathrm{p} / \mathrm{e}}$ for which a solution can be found for $M_{\mathrm{b}}=2.9 M_{\odot}$ and a given $\tilde{A}$ (i.e., the solver fails to converge when searching for a $M_{\mathrm{b}}=2.9 M_{\odot}$ mass model at densities outside the bounds of the symbols). The local minima of these curves are approximate turning points of the sequences. For the cold (c40p0) models, we have noted the range in $M_{\mathrm{g}}$ and $\rho_{\mathrm{b}, \max }$ across models with different amounts of differential rotation with dashed (solid) blue lines. Consequently, $\rho_{\mathrm{b}, \max }=1.30 \times 10^{15} \mathrm{~g} \mathrm{~cm}^{-3}$ represents the upper limit for the baryon density of a stable HMNS with the HShen EOS. Note also that the difference in $M_{\mathrm{g}}$ of the approximate turning points between sequences with the same temperature prescription is only $\sim 0.005 M_{\odot}$.

(A color version of this figure is available in the online journal.)

onset of instability for the equilibrium sequences for several reasons. (1) We find that the approximate turning points for all considered temperature parameterizations and measures of differential rotation $(\tilde{A}=0$ to $\tilde{A}=1.1$ with spacing $\delta \tilde{A}=0.1)$ lie within the same $\sim 25 \%$ range in $\rho_{\mathrm{b} \text {, max }}$ indicated by the blue lines in Figure 11 (similarly within a $\sim 25 \%$ range in $\rho_{\mathrm{b}, \max }$ for the LS220 EOS). (2) In cold uniformly rotating NS models, approximate turning points occur where one out of three of $d / d \rho_{\mathrm{b}, \max }\left(M_{\mathrm{g}}, M_{\mathrm{b}}, J\right)$ vanish. The study of such models shows that the actual turning point density is within only $\sim 1 \%$ of the approximate turning point density (where $d M_{\mathrm{g}} / d \rho_{\mathrm{b}, \max }=0$ along the mass-shed sequence; see Figure 10 of Stergioulas \& Friedman 1995). (3) The turning-point condition is a sufficient, but not necessary, criterion for secular instability. Thus instability must set in at $\rho_{\mathrm{b}, \max }$ greater than the turning-point $\rho_{\mathrm{b}, \max }$, but may set in already at lower densities (see, e.g., Takami et al. 2011 for an example). It is thus conservative to use the approximate turning point located at the highest $\rho_{\mathrm{b}, \max }$ over all sequences for a given EOS as an upper bound for the maximum stable $\rho_{\mathrm{b}, \max }$ of HMNS models for that EOS.

Further to the above, we have verified (see Section 9.1 of Kaplan 2014) that the same density ranges contain approximate turning points when examining alternate pairs of conserved variables: both $J$ and $M_{\mathrm{b}}$, and $J$ and $M_{\mathrm{g}}$ (in contrast to Figure 11, where we examine $M_{\mathrm{b}}$ and $M_{\mathrm{g}}$ ). This gives us confidence that the method of approximate turning points is self consistent with respect to choice of the vanishing derivatives. Unfortunately, since the CST code employs only barytropic EOSs, we lack the infrastructure necessary to study the total entropy of the configurations, and note that an examination of total entropy of these models is an important goal for future work.

\subsection{The Secular Evolution of HMNS from Mergers}

An HMNS remnant resulting from the merger of two NSs that does not promptly collapse into a black hole will settle into a quasiequilibrium state. More precisely, this is a state in which the HMNS is no longer in dynamical evolution, measured, for example, by oscillations in the HMNS maximum density. This should occur several dynamical times after merger. From this point on, the HMNS will evolve secularly along some sequence of equilibrium models. A secular evolution is, by definition, a dissipative process that may involve energy loss ${ }^{11}$ from the system. Consequently, we may parameterize the secular evolution of the HMNS toward a turning point via the change in its total mass-energy, which, in our case, is the change in gravitational mass of the equilibrium model. This occurs in HMNSs via neutrino cooling and the emission of gravitational radiation. In addition, the rotational energy of the HMNS may be reduced by angular momentum redistribution via the MRI, provided this occurs sufficiently slowly to be characterized as a secular process. This can lead to a build up of magnetic field, or dissipation of the free energy of differential rotation as heat (see, e.g., Thompson et al. 2005 for a detailed discussion), which may lead to increased neutrino cooling. Furthermore, specific angular momentum transported to the HMNS surface may unbind surface material, leading to a decrease in $J$ and $M_{\mathrm{b}}$. These changes of $M_{\mathrm{b}}$ and $J$ may be significant, but cannot be taken into account by the approximate description of the HMNS's evolution we are considering here. Our results should thus be interpreted with these limitations in mind.

A secularly evolving HMNS will, in general, evolve in the direction of decreasing gravitational mass $M_{\mathrm{g}}$ while (at least approximately) conserving its total baryonic mass $M_{\mathrm{b}}$. This results in an increasing density and compactness of the star. Figure 11 shows, for a fixed temperature prescription and differential rotation parameter, that the gravitational mass $M_{\mathrm{g}}$ of a sequence with fixed baryonic mass $M_{\mathrm{b}}=2.9 M_{\odot}$ (using the HShen EOS; we find qualitatively the same for the LS220) is decreasing with increasing density. This continues until, $M_{\mathrm{g}}$ reaches a minimum at an approximate turning point for $\rho_{\mathrm{b}, \text { max }} \gtrsim 1 \times 10^{15} \mathrm{~g} \mathrm{~cm}^{-3}$. Here, $\delta M_{\mathrm{g}}=0$, and $\delta M_{\mathrm{b}}$ vanishes by our choice of a constant $M_{\mathrm{b}}$ sequence.

The curves in Figure 11 are shown for constant differential rotation parameter $\tilde{A}$. However, an HMNS of $M_{\mathrm{b}}=2.9 M_{\odot}$ is not necessarily constrained to a specific curve. One would expect the HMNS to evolve to neighboring curves of less extreme differential rotation (decreasing $\tilde{A}$ ), in accordance with its loss of angular momentum due to gravitational waves and its redistribution of angular momentum due to other secular processes. Nevertheless, consider the limit in which the HMNS is constrained to a curve of constant $\tilde{A}$. Then it would evolve secularly until reaching the curve's minimum. At this point, any further energy loss implies that the HMNS must either (1) secularly evolve to a nearby equilibrium sequence with lower temperature or lower degree of differential rotation and higher density (another curve on the plot) or (2) undergo collapse to a black hole. Note that the densities at which the minimum occurs for different $\tilde{A}$ and temperatures are remarkably close

\footnotetext{
11 The trapped lepton number is, of course, also changing, since the fluxes of $\nu_{e}$ and $\bar{\nu}_{e}$ will at least initially not be symmetric. However the effect of the trapped lepton fraction on stability is minimal, since electron degeneracy pressure is present only at high densities where it is much smaller than the baryon pressure in hot HMNSs that lose energy to neutrino emission (see Figure 1).
} 
to each other. For the sequences using the HShen EOS shown in Figure 11, the approximate turning points lie in the range $1.05 \times 10^{15} \mathrm{~g} \mathrm{~cm}^{-3}<\rho_{\mathrm{b}, \max }<1.30 \times 10^{15} \mathrm{~g} \mathrm{~cm}^{-3}$ for all considered $\tilde{A}$ and both shown temperature prescriptions. The constant- $M_{\mathrm{b}}$ curves for other temperature parameterizations (c20p0, c30p0, c30p5, c30p10) are all located in-between the curves for the $\mathrm{c} 40 \mathrm{p} 0$ and cold cases shown. Thus, we expect that the point of collapse for an HMNS will be marked by its evolution to this density regime regardless of the temperature distribution of the model.

From the above findings, we conclude that thermal effects have little influence on the stability of HMNSs in rotational equilibrium against gravitational collapse. However, our results do imply that thermal support will affect at what density the HMNS first settles to its quasiequilibrium state. The discussion in Section 4.2 and, in particular, Figure 10, illustrates that at subcritical rotation rates and densities significantly below those of the approximate turning points, models with hot temperature profiles have a larger $M_{\mathrm{b}}$ compared to models with cooler temperatures at the same $\rho_{\mathrm{b}, \max }$. Thus an HMNS with greater thermal support will reach a quasiequilibrium at a lower $\rho_{\mathrm{b}, \max }$, and thus have more energy to lose before it can evolve to the critical density regime for collapse.

While thermal effects may be important in setting the initial conditions for the secular evolution of an HMNS, they appear to be of little consequence to the stability of an HMNS in quasiequilibrium. Once in a quasiequilibrium state, the energy lost by an HMNS during its secular evolution is the most robust indicator for its progress toward instability and collapse. Figure 11 shows that this is true regardless of the degree of differential rotation of the HMNS. For a fixed temperature parameterization, the difference in $M_{\mathrm{g}}$ between different degrees of differential rotation is at most $\sim 0.005 M_{\odot}$, corresponding to $\lesssim 10 \%$ of the total energy lost during the HMNS's secular evolution.

\subsection{Comparison with NSNS Merger Simulations}

Sekiguchi et al. (2011) conducted simulations of NSNS mergers using the HShen EOS and included neutrino cooling via an approximate leakage scheme. They considered three equalmass binaries with component NS gravitational (baryonic) masses of $1.35 M_{\odot}\left(1.45 M_{\odot}\right), 1.50 M_{\odot}\left(1.64 M_{\odot}\right), 1.60 M_{\odot}$ $\left(1.77 M_{\odot}\right)$ denoted as $\mathrm{L}, \mathrm{M}$, and $\mathrm{H}$, respectively. The HMNS formed from their high-mass binary collapses to a black hole within $\lesssim 9 \mathrm{~ms}$ of merger. The low-mass and the intermediatemass binaries, however, form hot $(T \sim 5-30 \mathrm{MeV})$ spheroidal quasiequilibrium HMNSs that remain stable for at least $25 \mathrm{~ms}$, the duration of their postmerger simulations.

Sekiguchi et al. (2011) argue that thermal pressure support could increase the maximum mass of HMNSs with $T \gtrsim 20 \mathrm{MeV}$ by $20 \%-30 \%$. The results that we lay out in Sections 3 and 4 of our study suggest that it is not straightforward to disentangle centrifugal and thermal effects for differentially rotating HMNS. Our findings show that critically spinning configurations (i.e., configurations at which the maximum $M_{\mathrm{b}}$ is obtained for a given $\tilde{A}$ ) of hot models do not lead to an increase in the maximum supported baryonic mass by more than a few percent and in most cases predict a lower maximum mass than in the cold case. We find it more useful to consider the results of Sekiguchi et al. (2011) in the context of the evolutionary scenario outlined in Section 5.2.

In Figure 12, we plot $M_{\mathrm{b}}$ as a function of $\rho_{\mathrm{b}, \max }$ for select sequences of uniformly and differentially rotating models obtained

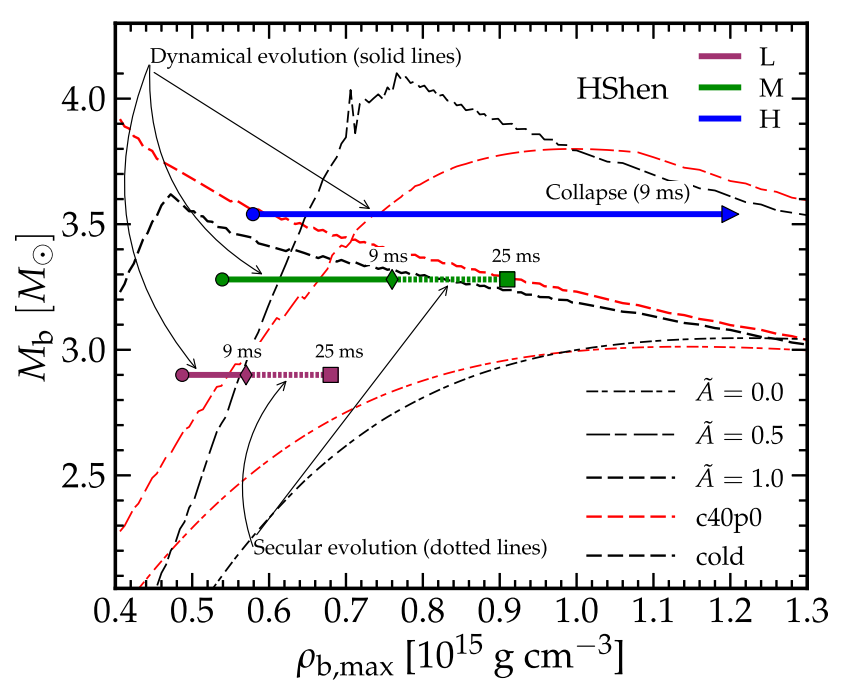

Figure 12. Similar to Figure 7 but for the HShen EOS and showing the approximate evolution of HMNSs from Sekiguchi et al. (2011). We show the evolution of maximum density of the HMNS for the low, medium and high mass configurations (thick lines L, M and $\mathrm{H}$ ) starting from the premerger density (noted by circles), and ending at the simulation termination densities (squares, or, in the $\mathrm{H}$ configuration, an arrow indicating collapse to a black hole). After $\sim 9 \mathrm{~ms}$ (noted with diamonds), the $\mathrm{L}$ and $\mathrm{M}$ models show negligible dynamical oscillations and have settled to a quasiequilibrium state. From there until the end of the simulation, the L and M HMNS are evolving secularly (indicated by thick dotted lines). We note that given the limitations of our approach discussed in the main text, the evolutionary tracks of constant baryonic mass shown in this figure should not be considered quantitatively reliable.

(A color version of this figure is available in the online journal.)

with the HShen EOS with the cold and c40p0 temperature prescriptions. We also mark the immediate postmerger densities of the L, M, and H models of Sekiguchi et al. (2011) and their evolutionary tracks (in $\rho_{\mathrm{b}, \max }$ ). The high-mass model $\mathrm{H}$ never settles into a quasiequilibrium and collapses to a black hole during the dynamical early postmerger phase. Its $\rho_{\mathrm{b}, \max }$ evolves within $\sim 9 \mathrm{~ms}$ from $0.58 \times 10^{15} \mathrm{~g} \mathrm{~cm}^{-3}$ to values beyond the range of the plot. Our secular-evolution approach cannot be applied to this model since it never reaches a quasiequilibrium state. The lower-mass $\mathrm{M}$ and $\mathrm{L}$ models enter Figure 12 at successively lower densities. Their "ring-down" oscillations are damped by $\sim 9 \mathrm{~ms}$ after which the HMNSs evolve secularly with $\rho_{\mathrm{b} \text {, max }}$ that increase roughly at the same rate in both models, suggesting that their rate of energy loss is comparable. At such early times, gravitational waves are most likely dominating energy loss (see the discussion of timescales in Paschalidis et al. 2012), and, indeed, model $\mathrm{M}$ and $\mathrm{L}$ exhibit similar gravitational wave amplitudes and frequencies (Sekiguchi et al. 2011, Figure 4). Focusing on model L, we now consider Figure 11, which shows sequences of constant $M_{\mathrm{b}}$ (for model L with $M_{\mathrm{b}} \sim 2.9 M_{\odot}$ ). As the HMNS loses energy, $M_{\mathrm{g}}$ decreases and the HMNS evolves to the right (toward higher $\rho_{\mathrm{b}, \max }$ ). Model L enters its secular evolution at a central density of $\sim 0.56 \times 10^{15} \mathrm{~g} \mathrm{~cm}^{-3}$ and evolves secularly to $\sim 0.68 \times 10^{15} \mathrm{~g} \mathrm{~cm}^{-3}$ within $\sim 16 \mathrm{~ms}$. Largely independent of its specific angular momentum distribution and thermal structure, Figure 11 suggests that this model will reach its global minimum $M_{\mathrm{g}}$ and, thus, instability in a small density range of $\sim 1.05-1.30 \times 10^{15} \mathrm{~g} \mathrm{~cm}^{-3}$.

Using our approximate secular evolution model for HMNSs discussed in Section 5.2, we linearly extrapolate the density evolution of model L in Sekiguchi et al. (2011). We expect a possible onset of collapse at $t \gtrsim 58 \mathrm{~ms}$ after merger (and 
$\gtrsim 49 \mathrm{~ms}$ after the start of the secular evolution). These numbers should be regarded as very rough estimates, given the limitations and rather qualitative nature of our model. Depending on its angular momentum when entering its secular evolution, its cooling rate, angular momentum redistribution and loss, model L may alternatively evolve into a long-term stable supramassive NS, since a baryonic mass of $\sim 2.9 M_{\odot}$ can in principle be supported by the HShen EOS at the supramassive limit (see Table 2). Furthermore, we have also checked that model L of Sekiguchi et al. (2011) contains sufficient angular momentum to be represented by the sequences identified in Figures 11 and 12. At a time of $\sim 10-15 \mathrm{~ms}$ after merger, model $\mathrm{L}$ has an angular momentum of $6 \times 10^{49} \mathrm{~g} \mathrm{~cm}^{2} \mathrm{~s}^{-1}$ (6.8 in $c=G=M_{\odot}$ units). Plots of similar sequences can be found in Kaplan (2014). They are consistent with this value.

The role of thermal pressure effects in all of the above is relatively minor (see the very similar $\rho_{\mathrm{b}, \max }$ locations of the $M_{\mathrm{g}}$ minima in hot and cold configurations shown in Figure 11). However, when first entering the secular regime as a subcritical HMNS, a configuration with higher temperature and stronger thermal pressure support will be less compact and will have a lower $\rho_{\mathrm{b}, \max }$ at a fixed $M_{\mathrm{b}}$ than a colder one. Hence, in the picture of secular HMNS evolution discussed in Section 5.2, such a configuration would have to evolve "farther" in $\rho_{\mathrm{b}, \max }$ to reach criticality and, thus, can survive longer at fixed energy loss rates.

Paschalidis et al. (2012) performed NSNS merger simulations of $\Gamma=2$ polytropes in which they approximated a thermal pressure component with a $\Gamma=2 \Gamma$-law. Their postmerger HMNS enters its secular evolution in a quasitoroidal configuration with two high-density, low-entropy cores, a central, lower-density, hot region and a high-entropy low-density envelope. The total mass of their model can be arbitrarily rescaled, but in order to estimate temperatures and thermal pressure contributions, the authors scaled their HMNS remnant to a gravitational mass of $2.69 M_{\odot}$. With this, they estimated in their quasitoroidal HMNS peak and rms temperatures of $\sim 20 \mathrm{MeV}$ and $\sim 5 \mathrm{MeV}$, respectively. Paschalidis et al. (2012) studied the effect of neutrino cooling on the HMNS evolution by introducing an ad-hoc cooling function that removes energy proportional to the thermal internal energy (neglecting the stiff temperature dependence of neutrino cooling). In order to capture effects of cooling during the limited simulated physical postmerger time, they drained energy from their HMNS at rates $\sim 100-200$ times higher than realistic cooling by neutrinos.

The authors considered cases without cooling and with two different accelerated cooling timescales. As cooling is turned on in their simulations, the slope of the maximum baryon density $\rho_{\mathrm{b}, \max }(t)$ of the HMNS increases discontinuously and the higher the cooling rate, the faster the evolution to higher $\rho_{\mathrm{b}, \max }(t)$. The HMNSs in both cases with cooling become unstable at different times, but roughly at the same $\rho_{\mathrm{b} \text {,max }}$. This is consistent with the secular HMNS evolution picture laid out in Section 5.2. Cooling reduces the total energy of the system $\left(M_{\mathrm{g}}\right)$ and drives the HMNS to higher $\rho_{\mathrm{b}, \max }$ at fixed $M_{\mathrm{b}}$ until the (approximate) turning point is reached and collapse ensues. However, losses due to gravitational wave emission and angular momentum redistribution and shedding will have the same effect and may dominate in nature, since they are likely to operate more rapidly than neutrino cooling (see the discussion of timescales by Paschalidis et al. 2012).

Bauswein et al. (2010) carried out smoothed-particle hydrodynamics simulations of HMNSs in the conformal-flatness approximation to general relativity. They compared simulations using the full temperature dependence of the HShen and LS180 EOS $^{12}$ with an approximate treatment of thermal pressure via a $\Gamma$-law, $P_{\text {th }}=\left(\Gamma_{\text {th }}-1\right) \epsilon_{\text {th }} \rho_{\mathrm{b}}$. Although Bauswein et al. (2010) do not provide a figure showing the evolution of maximum baryon density, they show (in their Figure 5) graphs of cumulative mass as a function of distance from the center of the LS180-EOS HMNS at $8 \mathrm{~ms}$ after merger, roughly the time when the dynamical early postmerger phase is over and the secular HMNS evolution begins. From this, it may be observed that the HMNS with the lower thermal $\Gamma\left(\Gamma_{\text {th }}=1.5\right)$ is more compact than the model with $\Gamma_{\text {th }}=2$. The HMNS evolved with the fully temperature-dependent LS180 EOS is in between the two, but closer to the $\Gamma_{\text {th }}=2$ model. Bauswein et al. (2010) found that the more compact HMNS with $\Gamma_{\text {th }}=1.5$ collapses after $10 \mathrm{~ms}$, while the less compact $\Gamma_{\text {th }}=2.0$ and full-LS180 cases collapse after $\sim 20 \mathrm{~ms}$. This is consistent with the picture of secular HMNS evolution drawn in Section 5.2. Given a fixed number of baryons, a less compact configuration has a lower maximum baryon density after merger and, therefore, begins its secular evolution (in the sense of Figures 11 and 12) at a lower density than a more compact configuration. Consequently, it must lose more energy before reaching the critical density for collapse.

The above illustrates how thermal pressure effects may increase the lifetime of an HMNS by affecting the initial conditions for its secular evolution. From Section 4 one notes that hot configurations, at densities below $\lesssim 10^{15} \mathrm{~g} \mathrm{~cm}^{-3}$ (the exact value being EOS dependent), may support significantly larger masses than their cold counterparts at the same $\rho_{\mathrm{b}, \max }$. Thus, during the dynamical settle-down of two merging NSs to a secularly evolving HMNS remnant, a configuration with lower thermal pressure will need to evolve to higher $\rho_{\mathrm{b}, \max }$ to reach an equilibrium configuration.

\section{SUMMARY AND CONCLUSIONS}

The merger of double NSs with component masses in the most commonly observed mass range $\left(\sim 1.3-1.4 M_{\odot}\right.$; Lattimer 2012$)$ is most likely to result in a hot, differentially spinning HMNS remnant that is stable against collapse on a dynamical timescale, but likely secularly evolving toward instability, driven by energy loss. While a number of merger simulations in approximate or full general-relativity with the necessary microphysics are now available, the role of thermal pressure support on the postmerger HMNS and its stability is not well understood.

In this study, we have attempted to gain insight into the role of thermal pressure support by constructing nonrotating, uniformly rotating and differentially rotating axisymmetric equilibrium solutions with multiple microphysical, fully temperature and composition dependent EOS and parameterized temperature distributions motivated by results from full merger simulations. Such axisymmetric equilibrium models may be acceptable approximations to merger remnants that have survived the initial highly dynamical and strongly nonaxisymmetric postmerger evolution and have settled down into longer-term stable quasiequilibrium. How far away the equilibrium configurations really are from real HMNSs, and the reliability of our results, will ultimately have to be established by more detailed comparisons with merger simulations in future work.

In the secular postmerger phase, the baryonic mass $M_{\mathrm{b}}$ of the hypermassive merger remnant is approximately conserved. 12 The LS180 is the variant of the Lattimer \& Swesty (1991) EOS with
nuclear compressibility modulus $K_{0}=180 \mathrm{MeV}$. 
Thus the dependence of the maximum of $M_{\mathrm{b}}$ on temperature is the most interesting quantity to study. In spherical symmetry (the TOV case), we find that at densities significantly lower than the density at which the maximum mass configuration occurs, thermal enhancement of the NS mass can be strong. Generally, hotter configurations yield the same $M_{\mathrm{b}}$ at lower central densities than their colder counterparts. However, when considering compact maximum- $M_{\mathrm{b}}$ configurations, thermal effects are small. For reasonable temperature prescriptions, hot temperatures lead to a small $(\lesssim 1 \%)$ decrease of $M_{\mathrm{b}}^{\max }$ for five out of the seven EOS that we consider. The two other EOS, the HShen EOS and the GShen-FSU2.1 EOS, show up to $~ 2 \%$ thermal enhancement of $M_{\mathrm{b}}$. As expected, none of the considered EOS could support a remnant of the merger of a canonical double NS system with typical masses.

Rapidly uniformly spinning configurations can support supramassive NSs. We have studied uniformly spinning sequences generated with the LS220 and HShen EOS. As in the TOV case, we find significant thermal enhancement of $M_{\mathrm{b}}$ at low central densities and rotation rates up to mass shedding. At high densities, however, thermal pressure is much less important for the support of the inner NS core, but bloats the envelope. This results in hotter configurations reaching mass shedding at lower angular velocities than colder configurations. Hence, at the mass-shedding supramassive limit, $M_{\mathrm{b}}$ and $M_{\mathrm{g}}$ decrease with increasing temperature for uniformly spinning NSs. For the LS220 EOS (HShen EOS), the cold supramassive $M_{\mathrm{b}}$ limit is $\sim 2.823 M_{\odot}\left(\sim 3.046 M_{\odot}\right)$. Under the plausible assumption that the HMNS merger remnant evolves toward a uniformly rotating configuration, assuming no mass loss during or after merger, the cold supramassive limit corresponds to component gravitational masses in an equal-mass progenitor binary of $M_{\mathrm{g}} \sim 1.287 M_{\odot}\left(M_{\mathrm{g}} \sim 1.403 M_{\odot}\right)$. On the other hand, a supramassive LS220 (HShen) NS with a $30 \mathrm{MeV}$ core and a $10 \mathrm{MeV}$ envelope has a supramassive limit $M_{\mathrm{b}} \sim 2.587 M_{\odot}$ $\left(M_{\mathrm{b}} \sim 2.808 M_{\odot}\right)$, which corresponds to binary component $M_{\mathrm{g}} \sim 1.185 M_{\odot}\left(M_{\mathrm{b}} \sim 1.300 M_{\odot}\right)$. Hence, cold maximally uniformly rotating configurations of LS220 and HShen NSs may barely support the merger remnant of canonical double NS binaries, but hot ones might not.

Differential rotation adds yet another layer of complexity, but is the most interesting scenario, since hypermassive merger remnants are born with differential rotation. The notion of a maximum mass of a differentially rotating HMNS is somewhat misleading, since different rotation laws will give different masses and different solvers may converge to different branches in the solution space. Hence, all "maximum" masses quoted are lower limits. For the commonly used $j-$ const. rotation-law, parameterized by the dimensionless parameter $\tilde{A}$, we find $M_{\mathrm{b}}$ up to $\sim 3.65 M_{\odot}$ and $\sim 4.10 M_{\odot}$, for the LS220 EOS and the HShen EOS, respectively. These high-mass configurations generally occur at densities that are up to a factor of two lower than those of maximum- $M_{\mathrm{b}}$ TOV and uniformly rotating models. Even higher masses could be found, but such configurations would be dynamically nonaxisymmetrically unstable.

Our results indicate that the role of thermal effects depends very much on the degree of differential rotation in addition to maximum density and (central) angular velocity. All qualitative findings are identical for the LS220 EOS and the HShen EOS. For critically rotating models (with minimum axis ratio $r_{\mathrm{p} / \mathrm{e}}$ for which a solution is found) the dependence on differential rotation is as follows. (1) For a low degree of differential rotation $(\tilde{A} \lesssim 0.4)$, the same systematics as found for the uniformly rotating case hold. (2) In models with intermediate degree of differential rotation $(\tilde{A} \sim 0.5-0.7)$, hot configurations have systematically lower "maximum" $M_{\mathrm{b}}$ than colder ones. (3) Models with high degree of differential rotation $(\tilde{A} \gtrsim 0.7)$ are mostly quasitoroidal and the "maximum" $M_{\mathrm{b}}$ occurs at low densities $\left(\lesssim 5 \times 10^{14} \mathrm{~g} \mathrm{~cm}^{-3}\right)$ and is mildly enhanced by thermal pressure support for models with hot cores, but cold envelopes. Models with high-temperature envelopes remain spheroidal until higher densities and have lower "maximum" $M_{\mathrm{b}}$. The situation is yet different for differentially rotating configurations that are rotating rapidly, but subcritically. For example, for LS220 EOS configurations with $\tilde{A}=1$, models with thermally supported envelopes have the highest $M_{\mathrm{b}}$ at subcritical rotation, but their sequences terminate at lower angular velocities (higher $\left.r_{\mathrm{p} / \mathrm{e}}\right)$ than the cold configuration, which ultimately catches up in $M_{\mathrm{b}}$ at critical rotation.

To summarize all of the above: the forecast is mixed-the role of thermal effects on the baryonic mass that is supported by a given configuration depends sensitively and in a complicated way on its details, that is, central/mean baryon density, temperature distribution, degree of differential rotation and rotation rate, to name the most important parameters. Configurations that yield "maximum" $M_{\mathrm{b}}$ are essentially unaffected by thermal effects. Beyond that, no simple general statements can be made.

A more useful way to reason about the role of thermal pressure support is to consider evolutionary sequences of equilibrium models representing the secular quasiequilibrium evolution of an HMNS. This evolution occurs along tracks of approximately constant baryonic mass $M_{\mathrm{b}}$ parameterized by maximum baryon density $\rho_{\mathrm{b}, \max }$. Since energy is lost by gravitational wave and neutrino emission, a configuration always evolves into the direction of decreasing total energy (i.e., decreasing gravitational mass $M_{\mathrm{g}}$ and increasing $\left.\rho_{\mathrm{b}, \max }\right)$. The turning point theorem (Sorkin 1982; Friedman \& Stergioulas 2013) says that an extremum in $M_{\mathrm{g}}$ may mark the point at which the sequence becomes secularly unstable to collapse. While this can be proven rigorously only for uniformly rotating (or nonrotating) configurations, we conjecture that it also holds at least approximately for the much more complex HMNS case. Provided this is true, we can define approximate turning points using constant- $M_{\mathrm{b}}$ sequences with different degrees of differential rotation and temperature parameterizations. With this, we find that the approximate turning points for a given $M_{\mathrm{b}}$ always lie in narrow ranges of $\rho_{\mathrm{b}, \max }$ and $M_{\mathrm{g}}$, which define the $M_{\mathrm{g}}-\rho_{\mathrm{b} \text {,max }}$ space in which collapse to a black hole occurs. Furthermore, the approximate turning point density at which collapse must set in depends only very weakly on temperature. Finally, we note that all approximate turning points found in this work are at baryon densities below the critical value for stable TOV stars. This may suggest that HMNS with maximum densities at or higher than the critical TOV central density could always be unstable to collapse. This possibility should be investigated further in future work.

Under the assumptions of the model laid out in this paper, the secular evolution of an HMNS can then be described by the progressive decrease of its gravitational mass $M_{\mathrm{g}}$ and increase of its maximum density $\rho_{\mathrm{b} \text {, max }}$. Our results show that an HMNS with more thermal pressure support will enter its secular evolution at a higher $M_{\mathrm{g}}$ and lower $\rho_{\mathrm{b}, \max }$ than a colder one (with the same rotational setup). Hence, the hot HMNS will have to evolve further in $\rho_{\mathrm{b}, \max }$ until reaching its approximate turning point. This explains the effects of thermal pressure observed in merger simulations (e.g., Bauswein et al. 2010; Sekiguchi et al. 
2011). We note that the same argument may also be applied to differences in HMNS spin: a more rapidly spinning HMNS will enter its secular evolution at lower $\rho_{\mathrm{b}, \max }$ and higher total energy and, hence, will have to evolve further in $\rho_{\mathrm{b}, \max }$ to reach its approximate turning point.

The goal of the work presented in this paper was to elucidate the role of thermal pressure support in hypermassive NSNS merger remnants on the basis of stationary spherically symmetric and axisymmetric equilibrium solutions of the Einstein-Euler equations. While yielding new insights, our present approach is limited in multiple ways. (1) Even in the secular quasiequilibrium evolution phase, HMNS are not exactly axisymmetric. The CST solver used in this study does not support nonaxisymmetric configurations, which makes it impossible for us to test how sensitive our results are to symmetry assumptions. (2) The equilibrium sequences considered here rely on an ad-hoc rotation law and ad-hoc temperature and composition parameterizations motivated by the simulations of Sekiguchi et al. (2011). In general, the angular velocity distribution will be more complex (see, e.g., Galeazzi et al. 2012) and the temperature and composition of an HMNS will not be single-parameter functions of density. (3) The CST solver has difficulties converging for configurations with a high degree of differential rotation and it is not clear if the terminating axis ratio $r_{\mathrm{p} / \mathrm{e}}$ is set by the formulation and implementation of the equations by the CST solver or if the termination occurs for physical reasons. This could be checked only by a comparison study with a more robust solver, e.g., the one of Ansorg et al. (2003). (4) The approximate turning point theorem that we have used to reason about the evolution and stability of HMNSs is heuristic and lacks rigorous foundation. Fully reliable statements about the stability of differentially rotating HMNSs with complex temperature and compositional distributions will require at least perturbative stability analysis or direct non-linear simulation.

Future work should address the above limitations (1)-(4) and should also consider rotating configurations constructed with a broader set of finite-temperature microphysical EOS.

We thank Eliot Quataert for inspiration and acknowledge helpful discussions with Lars Bildsten, Ursula C. T. Gamma, Jim Lattimer, Lee Lindblom, Sterl Phinney, Jocelyn Read, Yuichiro Sekiguchi, Masaru Shibata, Saul Teukolsky, Kip Thorne, and especially Aaron Zimmerman. Furthermore, we thank the anonymous referee for suggestions that improved this paper. This work was initiated at a Palomar Transient Factory Theory Network meeting at the Sky House, Los Osos, CA. C.D.O. thanks the Yukawa Institute for Theoretical Physics for hospitality during the long-term workshop Gravitational Waves and Numerical Relativity 2013 when this work was completed. This research is supported in part by NASA under the Astrophysics Theory grant No. NNX11AC37G, by the National Science Foundation under grant Nos. AST-1205732, PHY-1151197, AST-1212170, PHY-1068881, and PHY1068243 , by the Alfred P. Sloan Foundation and by the Sherman Fairchild Foundation. The calculations underlying the results presented in this paper were performed on the Caltech compute cluster "Zwicky" (NSF MRI award No. PHY-0960291). The EOS tables, driver, and TOV solver routines used in this work are available for download at http://www.stellarcollapse.org. The solver for axisymmetric equilibrium configurations of rotating HMNSs is not open source, but a similar solver may be obtained from http://www.lorene.obspm.fr/. The figures in this paper were generated with the matplotlib library for Python (Hunter 2007). This work was supported by Grant-in-Aid for Scientific Research (25103510, 25105508, 24740163), by HPCI Strategic Program of Japanese MEXT. The simulations were performed on XC30 at CfCA of NAOJ and SR16000 at YITP of Kyoto University.

\section{APPENDIX A}

\section{TEMPERATURE PARAMETERIZATIONS}

We consider temperature prescriptions with only a hot core at and above nuclear density and with a hot core and a more extended high-density plateau at lower densities. We emphasize that these prescriptions are rather ad-hoc and motivated primarily by the data from the simulations of Sekiguchi et al. (2011). All high-temperature regions are smoothly tapered-off ("rolled-off") using tanh functions.

The prescriptions with only a hot core (i.e., prescriptions cXp0) are given by

$$
\begin{array}{r}
T_{\text {roll }}\left(\rho_{\mathrm{b}} ; T_{1}, T_{2}, m, s\right)=T_{2}+\frac{\left(T_{1}-T_{2}\right)}{2} \\
\times\left(\tanh \frac{\left(\log _{10}\left(\rho_{\mathrm{b}}\right)-m\right)}{s}+1\right),
\end{array}
$$

where $m$ is the roll-off midpoint (in $\log _{10}\left(\rho_{\mathrm{b}}\left(\mathrm{g} \mathrm{cm}^{-3}\right)\right)$ and $s$ is the roll-off $e$-folding scale (also in $\log _{10}\left(\rho_{\mathrm{b}}\left(\mathrm{g} \mathrm{cm}^{-3}\right)\right)$. For prescriptions that only have hot cores, $T_{1}$ is set to the peak temperature $T_{\max }$ and $T_{2}$ is set to $T_{\min }=0.01 \mathrm{MeV}$. The prescriptions with a high-temperature plateau at lower densities, i.e., c30p5 and c30p10, are constructed as the sum of two of the above functions as follows:

$$
\begin{aligned}
& T\left(\rho_{\mathrm{b}} ; T_{\max }, T_{\min }, T_{p}, m^{\prime}, s^{\prime}\right)=T_{\min } \\
& \quad+T_{\text {roll }}\left(\rho_{\mathrm{b}} ; T_{1}=T_{p}, T_{2}=0, m=11.5, s=0.25\right) \\
& \quad+T_{\text {roll }}\left(\rho_{\mathrm{b}} ; T_{1}=T_{\max }-T_{p}, T_{2}=0, m^{\prime}, s^{\prime}\right)
\end{aligned}
$$

where $m^{\prime}$ is the roll-off midpoint, $s^{\prime}$ is the roll-off scale, and $T_{p}$ is the plateau temperature. Writing this out more explicitly, we have

$$
\begin{aligned}
T\left(\rho_{\mathrm{b}} ;\right. & \left.T_{\max }, T_{\min }, T_{p}, m, s\right)=T_{\min } \\
& +\frac{T_{p}}{2}\left(\tanh \frac{\left(\log _{10}\left(\rho_{\mathrm{b}}\right)-11.5\right)}{0.25}+1\right) \\
& +\frac{T_{\max }-T_{p}}{2}\left(\tanh \frac{\left(\log 10\left(\rho_{\mathrm{b}}\right)-m\right)}{s}+1\right) .
\end{aligned}
$$

Table 4 summarizes the parameters for generating the temperature prescriptions used in this study.

\section{APPENDIX B}

\section{SOLVING FOR THE ELECTRON FRACTION}

For a given EOS and temperature prescription, we find the electron fraction $Y_{e}$ by first solving for $Y_{e}$ assuming neutrinoless $\beta$-equilibrium for the cold case ( $T=0.01 \mathrm{MeV}$ or the lowest temperature point available in the EOS table), using the condition

$$
\mu_{v}=0=\mu_{n}+\mu_{p}-\mu_{e}
$$

for the chemical potentials. In the absence of neutrinos, the lepton fraction $Y_{\text {lep }}=Y_{e}$. In the hot case, neutrinos are trapped 
Table 4

Temperature Prescription Parameters

\begin{tabular}{lcrcc}
\hline \hline Model & $\begin{array}{c}T_{\max } \\
(\mathrm{MeV})\end{array}$ & $\begin{array}{c}\text { Midpoint } m \\
\log _{10}\left(\rho_{\mathrm{b}}\left(\mathrm{g} \mathrm{cm}^{-3}\right)\right)\end{array}$ & $\begin{array}{c}\text { Scale } s \\
\log _{10}\left(\rho_{\mathrm{b}}\left(\mathrm{g} \mathrm{cm}^{-3}\right)\right)\end{array}$ & $\begin{array}{c}\text { Plateau } \\
\text { Temperature } T_{p} \\
(\mathrm{MeV})\end{array}$ \\
\hline cold & $\ldots$ & $\ldots$ & $\ldots$ & $\ldots$ \\
c20p0 & 20 & $14.0-0.07$ & 0.25 & 0 \\
c30p0 & 30 & $14.125-0.07$ & 0.375 & 0 \\
c30p5 & 30 & $14.1875-0.07$ & 0.3125 & 5 \\
c30p10 & 30 & $14.25-0.07$ & 0.25 & 10 \\
c40p0 & 40 & $14.25-0.07$ & 0.5 & 0 \\
\hline
\end{tabular}

Notes. Parameters used for the temperature parameterizations used in this study. The notation is $\mathrm{c}\langle$ core temperature $\rangle \mathrm{p}\langle$ plateau temperature $\rangle$. All low-density temperature plateaus are tapered off at densities below $\sim 10^{12} \mathrm{~g} \mathrm{~cm}^{-3}$ with a tanh function with a midpoint at $\log _{10}\left(\rho_{\mathrm{b}}\left(\mathrm{g} \mathrm{cm}^{-3}\right)\right)=11.5$ and an $e$-folding width of $\log _{10}\left(\rho_{\mathrm{b}}\left(\mathrm{g} \mathrm{cm}^{-3}\right)\right)=0.25$. All minimum temperatures are $0.01 \mathrm{MeV}$. See Figure 2 for a comparison of the various temperature prescriptions. The functional form of the prescriptions is given by Equations (A1) and (A3).

in the HMNS matter above $\rho=\rho_{\text {trap }} \approx 10^{12.5} \mathrm{~g} \mathrm{~cm}^{-3}$ and $Y_{\text {lep }}=Y_{e}+Y_{v}$, where $Y_{v}=Y_{v_{e}}-Y_{\bar{v}_{e}}$.

We then take $Y_{\text {lep }}$ and solve for $Y_{e}$ in the hot case with neutrinos by treating the latter as a relativistic Fermi gas in equilibrium for which $Y_{\nu}$ can be calculated from the neutrino number density $n_{v}=n_{v_{e}}-n_{\bar{v}_{e}}$ via

$$
Y_{v}=\frac{n_{v}}{\rho N_{A}} .
$$

The neutrino number density is

$$
n_{v}=4 \pi\left(\frac{k_{B} T}{h c}\right)^{3}\left[F_{2}\left(\eta_{v}\right)-F_{2}\left(-\eta_{v}\right)\right]
$$

where $\eta_{v}=\mu_{\nu} /\left(k_{B} T\right)$ is the neutrino degeneracy parameter (Bludman \& van Riper 1978). Note that in equilibrium, $v_{e}$ and $\bar{v}_{e}$ have equal and opposite chemical potentials. $F_{2}$ is a Fermi integral given by

$$
F_{k}(\eta)=\int_{0}^{\infty} \frac{x^{k} d x}{e^{x-\eta}+1}
$$

In practice, we use

$$
F_{2}(\eta)-F_{2}(-\eta)=\frac{1}{3} \eta\left(\eta^{2}+\pi^{2}\right),
$$

which is given in Bludman \& van Riper (1978) and is exact for any degeneracy parameter $\eta$.

We find $Y_{e}$ by finding the root

$$
0=Y_{\text {lep }}-\left(Y_{e}+Y_{v}\right) \text {. }
$$

$Y_{\text {lep }}$ is a fixed input. We set $Y_{e}=Y_{\text {lep }}$ as an initial guess and $Y_{v}$ is calculated using Equations (B2), (B3), and (B5), with $\mu_{v}=\mu_{n}+\mu_{p}-\mu_{e}$ obtained from the EOS. $Y_{e}$ is then adjusted and we iterate until convergence.

Since neutrinos begin to stream freely below $\rho_{\text {trap }}$, we also compute $Y_{e}$ using the $\nu$-less $\beta$-equilibrium condition (Equation (B1)). We then compute a final effective $Y_{e}$ using

$$
\begin{aligned}
Y_{e, \text { eff }}(\rho, T[\rho])= & Y_{e, v-\operatorname{less} \beta}(\rho, T[\rho]) \times\left(1-e^{-\rho_{\text {trap }} / \rho}\right) \\
& +Y_{e, \beta}(\rho, T[\rho]) \times e^{-\rho_{\text {trap }} / \rho} .
\end{aligned}
$$

\section{REFERENCES}

Accadia, T., Acernese, F., Antonucci, F., \& Virgo Collaboration. 2011, CQGra, 28,114002

Ansorg, M., Kleinwächter, A., \& Meinel, R. 2003, A\&A, 405, 711

Antoniadis, J., Freire, P. C. C., Wex, N., et al. 2013, Sci, 340, 448

Baiotti, L., Giacomazzo, B., \& Rezzolla, L. 2008, PhRvD, 78, 084033

Baiotti, L., Pietri, R. D., Manca, G. M., \& Rezzolla, L. 2007, PhRvD, 75,044023

Baumgarte, T. W., Shapiro, S. L., \& Shibata, M. 2000, ApJL, 528, L29

Bauswein, A., Janka, H.-T., Hebeler, K., \& Schwenk, A. 2012, PhRvD, 86, 063001

Bauswein, A., Janka, H.-T., \& Oechslin, R. 2010, PhRvD, 82, 084043

Bludman, S. A., \& van Riper, K. A. 1978, ApJ, 224, 631

Chandrasekhar, S. 1969, Ellipsoidal Figures of Equilibrium (New Haven, CT: Yale Univ. Press), revised edition 1987

Cook, G. B., Shapiro, S. L., \& Teukolsky, S. A. 1992, ApJ, 398, 203

Cook, G. B., Shapiro, S. L., \& Teukolsky, S. A. 1994a, ApJ, 422, 227

Cook, G. B., Shapiro, S. L., \& Teukolsky, S. A. 1994b, ApJL, 423, L117

Corvino, G., Rezzolla, L., Bernuzzi, S., De Pietri, R., \& Giacomazzo, B. 2010, CQGra, 27, 114104

Demorest, P. B., Pennucci, T., Ransom, S. M., Roberts, M. S. E., \& Hessels, J. W. T. 2010, Natur, 467, 1081

Dessart, L., Ott, C. D., Burrows, A., Rosswog, S., \& Livne, E. 2009, ApJ, 690, 1681

Faber, J. A., \& Rasio, F. A. 2012, LRR, 15, 8

Friedman, B., \& Pandharipande, V. R. 1981, NuPhA, 361, 502

Friedman, J. L., \& Ipser, J. R. 1987, ApJ, 314, 594

Friedman, J. L., Ipser, J. R., \& Sorkin, R. D. 1988, ApJ, 325, 722

Friedman, J. L., Parker, L., \& Ipser, J. R. 1986, ApJ, 304, 115

Friedman, J. L., \& Stergioulas, N. 2013, Rotating Relativistic Stars (Cambridge: Cambridge Univ. Press)

Galeazzi, F., Yoshida, S., \& Eriguchi, Y. 2012, A\&A, 541, A156

Goussard, J. O., Haensel, P., \& Zdunik, J. L. 1997, A\&A, 321, 822

Goussard, J.-O., Haensel, P., \& Zdunik, J. L. 1998, A\&A, 330, 1005

Harry, G. M., \& LIGO Scientific Collaboration. 2010, CQGra, 27, 084006

Hempel, M., Fischer, T., Schaffner-Bielich, J., \& Liebendörfer, M. 2012, ApJ, 748,70

Hempel, M., \& Schaffner-Bielich, J. 2010, NuPhA, 837, 210

Hunter, J. D. 2007, CSE, 9, 90

Kaplan, J. D. 2014, PhD thesis, California Institute of Technology, Pasadena, CA

Kiuchi, K., Sekiguchi, Y., Shibata, M., \& Taniguchi, K. 2009, PhRvD, 80, 064037

Kochanek, C. S. 1992, ApJ, 398, 234

Komatsu, H., Eriguchi, Y., \& Hachisu, I. 1989a, MNRAS, 237, 355

Komatsu, H., Eriguchi, Y., \& Hachisu, I. 1989b, MNRAS, 239, 153

Lai, D. 1994, MNRAS, 270, 611

Lattimer, J. M. 2012, ARNPS, 62, 485

Lattimer, J. M., \& Prakash, M. 2001, ApJ, 550, 426

Lattimer, J. M., \& Prakash, M. 2007, PhR, 442, 109

Lattimer, J. M., \& Swesty, F. D. 1991, NuPhA, 535, 331

Liebendörfer, M. 2005, ApJ, 633, 1042

Morrison, I. A., Baumgarte, T. W., \& Shapiro, S. L. 2004, ApJ, 610, 941

Nakar, E. 2007, PhR, 442, 166

O'Connor, E., \& Ott, C. D. 2011, ApJ, 730, 70

Oechslin, R., Janka, H.-T., \& Marek, A. 2007, A\&A, 467, 395

Ostriker, J. P., Bodenheimer, P., \& Lynden-Bell, D. 1966, PhRvL, 17, 816

Ott, C. D., Dimmelmeier, H., Marek, A., et al. 2007, PhRvL, 98, 261101

Paschalidis, V., Etienne, Z. B., \& Shapiro, S. L. 2012, PhRvD, 74, 064032

Penner, A. J., Andersson, N., Jones, D. I., Samuelsson, L., \& Hawke, I. 2012, ApJL, 749, L36

Prakash, M., Lattimer, J. M., Pons, J. A., Steiner, A. W., \& Reddy, S. 2001, in Lecture Notes in Physics, Vol. 578, Physics of Neutron Star Interiors, ed. D. Blaschke, N. K. Glendenning, \& A. Sedrakian (Berlin: Springer), 364

Read, J. S., Lackey, B. D., Owen, B. J., \& Friedman, J. L. 2009, PhRvD, 79, 124032

Rosswog, S., \& Liebendörfer, M. 2003, MNRAS, 342, 673

Ruffert, M., \& Janka, H.-T. 2001, A\&A, 380, 544

Sekiguchi, Y., Kiuchi, K., Kyutoku, K., \& Shibata, M. 2011, PhRvL, 107, 051102

Shapiro, L. S., \& Teukolsky, S. A. 1983, Black Holes, White Dwarfs and Neutron Stars (New York: Wiley)

Shen, G., Horowitz, C. J., \& O'Connor, E. 2011a, PhRvC, 83, 065808

Shen, G., Horowitz, C. J., \& Teige, S. 2011b, PhRvC, 83, 035802

Shen, H., Toki, H., Oyamatsu, K., \& Sumiyoshi, K. 2011c, ApJS, 197, 20 
Shibata, M., Taniguchi, K., \& Uryu, K. 2005, PhRvD, 71, 084021 Somiya, K., \& KAGRA Collaboration. 2012, CQGra, 29, 124007 Sorkin, R. D. 1982, ApJ, 257, 847

Steiner, A. W., Hempel, M., \& Fischer, T. 2013, ApJ, 774, 17

Stergioulas, N., \& Friedman, J. L. 1995, ApJ, 444, 306

Takami, K., Rezzolla, L., \& Yoshida, S. 2011, MNRAS, 416, L1

Tassoul, J.-L. 1978, Theory of Rotating Stars (Princeton, NJ: Princeton Univ. Press)
Thierfelder, M., Bernuzzi, S., \& Brügmann, B. 2011, PhRvD, 84, 044012

Thompson, T. A., Quataert, E., \& Burrows, A. 2005, ApJ, 620, 861

Timmes, F. X., \& Arnett, D. 1999, ApJS, 125, 277

Tsang, D., Read, J. S., Hinderer, T., Piro, A. L., \& Bondarescu, R. 2012, PhRvL, 108,011102

van Riper, K. A., \& Bludman, S. A. 1977, ApJ, 213, 239

Watts, A. L., Andersson, N., \& Jones, D. I. 2005, ApJL, 618, L37

Weinberg, N. N., Arras, P., \& Burkart, J. 2013, ApJ, 769, 121 